\title{
Wheel profile optimization on railway vehicles from the wear viewpoint
}

\author{
M. Ignesti, A. Innocenti, L. Marini, E. Meli*, A. Rindi, P. Toni \\ Department of Energy Engineering, University of Florence, Via S. Marta n. 3, 50139 Firenze, Italy
}

\section{A R T I C L E I N F O}

Available online 27 December 2012

Keywords:

Multibody modelling of railway vehicles

Wheel-rail contact

Wheel-rail wear modelling

Wheel-rail profile optimization

\begin{abstract}
A B S T R A C T
The modelling and the reduction of wear due to wheel-rail interaction is a fundamental aspect in the railway field, mainly correlated to running stability and safety, maintenance interventions and costs. In this work the authors present two innovative wheel profiles, specifically designed with the aim of improving the wear and stability behaviour of the standard ORE S1002 wheel profile matched with the UIC60 rail profile canted at $1 / 20 \mathrm{rad}$, which represents the wheel-rail combination adopted in Italian railway line.

The two wheel profiles, conventionally named CD1 and DR2, have been developed by the authors in collaboration with Trenitalia S.p.A. The CD1 wheel profile has been designed with the purpose of spreading the contact points in the flange zone on a larger area in order to reduce wear phenomena and having a constant equivalent conicity for small lateral displacements of the wheelset with respect to the centred position in the track. The DR2 wheel profile is instead designed in order to guarantee the same kinematic characteristics of the matching formed by ORE S1002 wheel profile and UIC60 rail profile with laying angle $\alpha_{p}$ equal to $1 / 40$ rad, widely common in European railways and characterized by good performances in both wear and kinematic behaviour.

Wheel profiles evolution has been calculated through a wear model developed and validated by the authors in previous works with experimental data relative to the Italian Aosta-Pre Saint Didier railway line. This model comprises two mutually interactive units: a vehicle model for the dynamic simulations and a model for the wear assessment. The whole model is based on a discrete process: each discrete step consists in one dynamic simulation and one profile update by means of the wear model while, within the discrete step, the profiles are supposed to be constant. The choice of an appropriate step is crucial in terms of precision and computational effort: the particular strategy adopted in the current work has been chosen for its capacity in representing the non-linear wear evolution and for the low computational time required.

In the present research the investigated trainset is the passenger vehicle ALSTOM ALn 501 "Minuetto", which is usually equipped with the standard ORE S1002 wheel profile and UIC60 rail profile canted at $1 / 20$ rad in Italian railways. The entire model has been simulated on a virtual track specifically developed to represent a statistical description of the whole Italian line. The data necessary to build the virtual track and the vehicle model were provided by Trenitalia S.p.A. and Rete Ferroviaria Italiana (RFI). Both the innovative wheel profiles developed in this research activity for the UIC60 rail with cant $1 / 20$ rad have proven to work fine in terms of resistance to wear if compared with the old ORE S1002 wheel profile.
\end{abstract}

(c) 2012 Elsevier Ltd. All rights reserved.

\section{Introduction}

Wear phenomena due to wheel-rail interaction represent a critical aspect in railway applications; in fact the consequent evolution of rail and wheel profiles involves serious effects on both dynamical and stability characteristics of vehicles. From a

\footnotetext{
* Corresponding author.

E-mail addresses: ignesti@mapp1.de.unifi.it (M. Ignesti), innocenti@mapp1.de.unifi.it (A. Innocenti), marini@mapp1.de.unifi.it (L. Marini), enrico.meli@unifi.it, meli@mapp1.de.unifi.it (E. Meli), rindi@mapp1.de.unifi.it (A. Rindi), toni@mapp1.de.unifi.it (P. Toni).
}

safety standpoint, modifications in wheel and rail profiles may compromise the vehicle stability and also increase the derailment risk due to wheels climbing over the rail. Profile changes lead also to higher maintenance cost, mainly concerned with the periodically re-profiling operations of wheels and the undesirable replacements of rails, necessary to re-establish the original profiles. A reliable wear model can be used to optimize the original profiles of wheel and rail and to obtain a more uniform wear on rolling surfaces. In such a way the overall amount of worn material can be reduced, the mean time between two maintenance interventions can be increased and, at the same time, the dynamical performance of the wheel-rail pair can be kept approximately 
constant between two succeeding repair interventions. The shape optimization of profiles for the reduction of wear at the wheelrail interface represents so an important aspect in railway field and various approaches were developed to obtain a satisfactory combination of wheel and rail profiles. The optimum matching is usually pursued through the design of a new wheel profile which matches an existing rail profile, because the cost of rail interventions is notably higher compared with the cost of turning or replacement of the wheels.

This paper describes the design procedure and the behaviour in wear reduction of two innovative wheel profiles, developed by the authors in collaboration with Trenitalia and RFI, with the aim of reducing wheel-rail wear that occurs when coupling ORE S1002 wheel profile and UIC60 rail profile with laying angle $\alpha_{p}$ equal to $1 / 20 \mathrm{rad}$, as it occurs in Italian line. The two wheel profiles proposed in this paper are conventionally named CD1 wheel profile and DR2 wheel profile [1].

CD1 wheel profile has been designed starting from two different purposes. The first consists in distributing the contact points in the flange zone on a larger area in order to reduce wear phenomena. The second purpose is based on having a constant equivalent conicity value in a band around the initial contact point (when the generical wheelset is not shifted from the central position).

The design procedure of DR2 wheel profile aims to keep with the new profile the kinematic characteristics of the matching formed by ORE S1002 wheel profile and UIC60 rail profile with laying angle $\alpha_{p}$ equal to 1/40 rad, widely common in European railways and characterized by good performances in both wear and kinematic behaviour.

The evolution of wheel profiles has been evaluated by means of a model specifically developed for the wear assessment, which has been validated by the authors in previous works [2-4] with experimental data relative to the Italian Aosta-Pre Saint Didier railway line.

The trainset to be investigated in order to evaluate the capability in wear reduction of the two innovative profiles is the passenger vehicle ALSTOM ALn 501 "Minuetto", which is usually equipped with the standard ORE S1002 wheel profile and UIC60 rail profile canted at $1 / 20$ rad in Italian railway. This particular vehicle exhibits in fact severe wear and stability problems mainly caused by the adopted matching.

All the simulations are performed on a virtual track, specifically designed to represent a statistical description of the whole Italian railway line [1]. This statistical railway line is a set of $N_{c}$ curvilinear track characterized, as it will be explained in the following sections, by specific radius $R$, superelevation $h$, velocity $V$ and statistical weight $p_{k}$ (with $1 \leq k \leq N_{c}$ ) values. A mean constant worn rail profile (in statistical sense) is then associated to each curvilinear track of the statistical railway line.

The data necessary to build the multibody model of the vehicle and the virtual track model were, respectively, provided by Trenitalia S.p.A and RFI [1].

\section{General architecture of the model}

More specifically the general architecture of the developed model comprises two mutually interactive parts: the vehicle model (multibody model and 3D global contact model) and the wear model (local contact model, wear estimation and profiles updating).

The general architecture of the model developed by the authors consists in a discrete procedure articulated in two separate parts that work alternatively at each procedure step: the vehicle model and the wear model. The general layout of the entire model is illustrated in the diagram in Fig. 1. The vehicle model is the part responsible for the dynamical simulations and it is based on the online mutual interaction of two submodels, namely the multibody model of the vehicle to be investigated (in this work the ALSTOM ALn 501 "Minuetto") and the 3D global contact model. More specifically, at each integration step during time-domain dynamic simulation, the multibody model evaluates the kinematic variables (position $\mathbf{G}_{\mathrm{w}}$, orientation $\mathbf{R}$ and their derivatives $\left.\dot{\mathbf{G}}_{\mathbf{w}}, \boldsymbol{\omega}\right)$ of each wheelset of the considered vehicle. These variables are then passed to the 3D global contact model, whose task

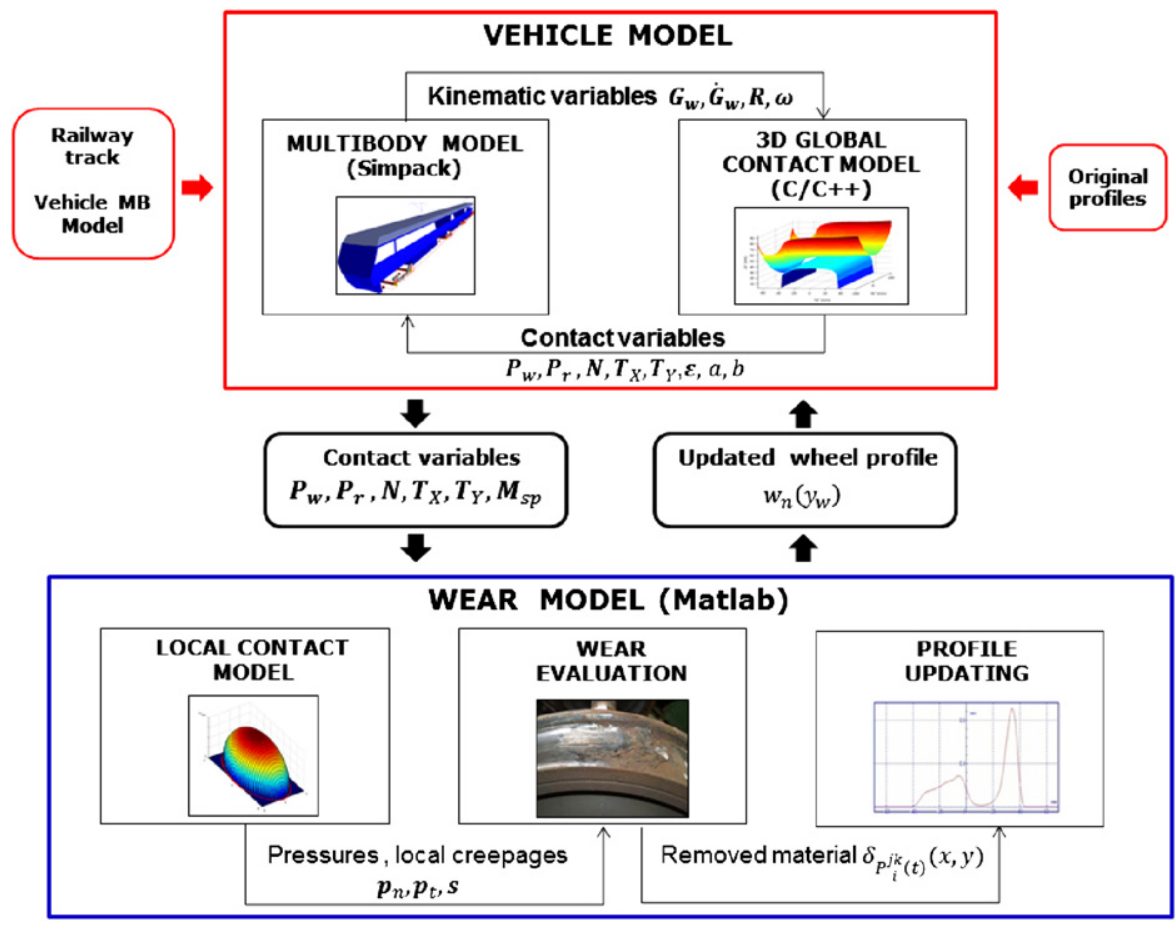

Fig. 1. General architecture of the model. 
consists in the calculation of the global contact parameters: contact points $\left(\mathbf{P}_{\mathbf{w}}, \mathbf{P}_{\mathbf{r}}\right)$, contact area dimensions $(a, b)$, global creepages $(\boldsymbol{\varepsilon})$ and contact forces $\left(\boldsymbol{N}^{r}, \mathbf{T}_{\mathbf{x}}^{\mathbf{r}}, \mathbf{T}_{\mathbf{y}}^{\mathbf{r}}\right)$. In particular, the contact points detection is based on an innovative algorithm developed by the authors in previous works [2-4], while the contact forces (normal and tangential forces) calculation is performed according to Hertz's and Kalker's global theories [5,6]. Once the tangential contact problem has been solved, the values of the global forces are sent back to the multibody model and the dynamical simulation proceeds with the next time integration step.

The inputs of the multibody model are the geometrical and inertial properties of the considered railway vehicle, the characteristics of the investigated track and the relative operating conditions. The geometrical inputs of the 3D global contact model are wheel and rail original profiles described by means of discrete sets of points. During this research project, according to the specifications required by Trenitalia, the capability on wear reduction of two innovative wheel profiles has been evaluated. Each of these profiles (CD1 and DR2 wheel profiles designed by the authors in collaboration with Trenitalia and RFI [1]) fits the considered vehicle in a specific simulation set.

The vehicle model for the simulations has been modelled in the commercial Multibody Software (MBS) Simpack. In particular, the multibody model has been defined in the Simpack Rail environment, while the wheel-rail 3D global contact model has been implemented in a subroutine written in C language. This subroutine is called by a specifically developed FORTRAN routine defined within the Simpack User routines module, which allows the customization of the contact model employed in the dynamical simulations.

The wear model is the part of the procedure concerning the computation of wheel profile evolution and it predicts the amount of worn material $\delta_{p^{i k}(t)}(x, y)$ to be removed from the wheel surfaces. The wear model can be subdivided into three parts: the local contact model, the evaluation of worn material and the profile updating. Firstly, the local contact model (whose approach is based on Hertz's local theory and Kalker's simplified theory implemented in FASTSIM algorithm) estimates the local contact pressures $\left(p_{n}, \mathbf{p}_{t}\right)$ and creepages (s) and detects the creep zone of the contact area. Then, according to an experimental relationship between the worn material and the energy dissipated by friction forces at the contact interface available in literature $[7,8]$, the quantity of removed material on wheel surface is computed on the creep area. This estimation is performed hypothesizing dry contact friction at the wheel-rail interface as Trenitalia and RFI requirements establish. Last step of the wear prediction procedure consists in updating the profiles: the worn profiles are derived from the original ones using an appropriate update strategy. The single mean profile $w_{n}(s)$ (the same one for all the vehicle) is then fed back as input to the entire vehicle model and the whole model procedure proceeds with the next discrete step.

In the present work the total mileage $\mathrm{km}_{\text {tot }}$ is subdivided in steps characterized by a variable adaptive length $\mathrm{km}_{\text {step }}$ and the wheel profile is supposed to be constant within each discrete step (corresponding to a travelled distance equal to $\mathrm{km}_{\text {step }}$ ). It is worth noticing that a decrease of the $k m_{\text {step }}$ value increases the model precision and, at the same time, the computational effort. The adopted updating strategy is a key point since it may appreciably affects the results and its main task consists in choosing the appropriate discrete steps for the update of wheel profiles. Two different strategies are available in literature, differing each other for the choice of the discrete step which has to consider the influence of the run distance by the investigated vehicle. These strategies are the constant step update strategy, which is characterized by a constant value $\mathrm{km}_{\text {step }}$ of the discrete step and the adaptive step update strategy, wherein the profile is updated when a given threshold of the maximum value of cumulative wear depth is reached and the value $\mathrm{km}_{\text {step }}$ is consequently variable.

During this research activity the adaptive step has been adopted for its capacity in representing the non-linear wear evolution (particularly in the first phase of the simulations, characterized by non-conformal wheel-rail contact). Furthermore, this strategy presents computational times comparable with those relative to the constant step update strategy.

The entire wear model has been implemented in the Matlab environment.

\section{The vehicle model}

The present section deals with the description of the vehicle model. First of all the multibody model for the dynamical simulations of the studied vehicle is introduced. Then the algorithm that models the 3D global contact is briefly explained.

The trainset investigated during this research is ALSTOM ALn 501 "Minuetto", a passenger transport unit widespread in Italian Railways, equipped with the standard ORE S1002 wheel profile and UIC60 rail profile canted at 1/20 rad. This particular vehicle exhibits in fact severe wear and stability problems mainly caused by the adopted matching. Its mechanical structure and inertial, elastic and damping properties can be found in literature [1]. Table 1 shows the main characteristics of the considered vehicle.

The multibody model of the vehicle has been implemented in the Simpack environment (see Fig. 2a) and it mainly consists of car bodies connected by means of the secondary suspensions to the vehicle bogies, which in turn are linked to wheelsets through the primary suspensions. Totally, the multibody model is made up by 31 rigid bodies:

- three coaches;

- four bogies: two external motor bogies and two intermediate trailer bogies interposed between two successive coaches;

- eight wheelsets: two for each bogie;

- sixteen axleboxes: two for each wheelset.

The inertial characteristics of the vehicle are listed in Table 2: it should be noticed that motors and gearboxes are not represented by an apposite body in the multibody model and their inertial properties have been included in the motor bogie frame and in the wheelset bodies.

The dual-stage suspensions have been modelled by means of three-dimensional linear and non-linear visco-elastic force elements. In the primary suspension stage the elastic elements are Flexicoil springs (constituted by two coaxial helical compression springs), while damping of the vertical relative displacement is provided by means of two non-linear dampers (see Fig. 2b).

Table 1

Main characteristics of the Aln 501 Minuetto DMU.

\begin{tabular}{ll}
\hline Length & $51.9 \mathrm{~m}$ \\
Width & $2.95 \mathrm{~m}$ \\
Height & $3.82 \mathrm{~m}$ \\
Bogie pivot distances & $14.8-13.8-14.8 \mathrm{~m}$ \\
Bogie wheelbase & $2.80 \mathrm{~m}$ \\
Unladen weight & $100 \mathrm{t}$ \\
Wheel arrangement & Bo-2-2-Bo \\
Wheel diameter & $850 \mathrm{~mm}$ \\
Max speed & $130 \mathrm{~km} / \mathrm{h}$ \\
\hline
\end{tabular}



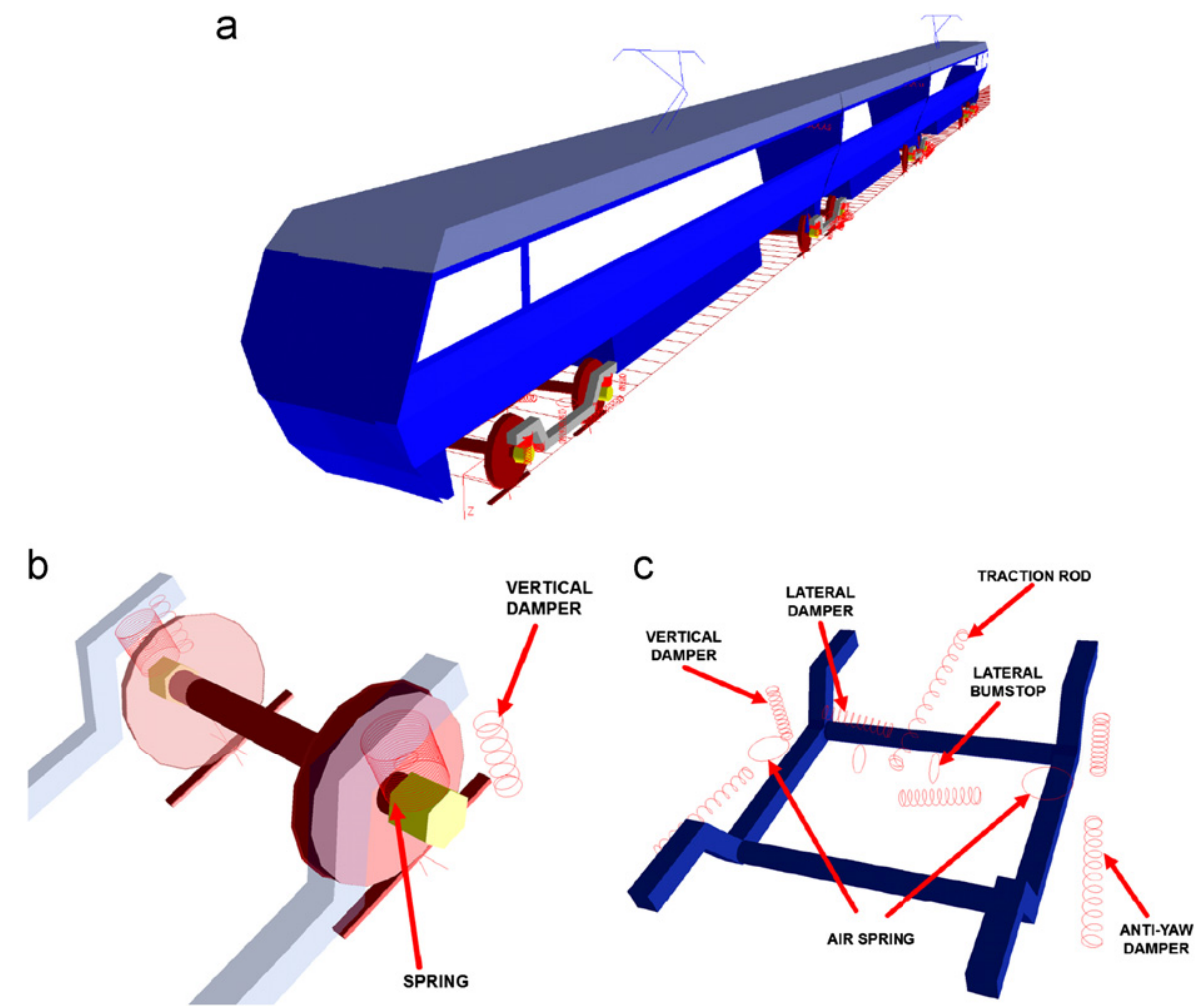

Fig. 2. Multibody model of the vehicle: (a) global view, (b) primary suspensions, (c) secondary suspensions.

Table 2

The Aln 501 Minuetto main inertial properties.

\begin{tabular}{lrrrrl}
\hline Body & Mass $(\mathrm{kg})$ & $I_{x x}\left(\mathrm{~kg} \mathrm{~m}^{2}\right)$ & $I_{y y}\left(\mathrm{~kg} \mathrm{~m}^{2}\right)$ & $I_{z z}\left(\mathrm{~kg} \mathrm{~m}^{2}\right)$ & $z_{C o G}(\mathrm{~m})$ \\
\hline External coach & 31568 & 66700 & 764000 & 743000 & -1.91 \\
Internal coach & 14496 & 30600 & 245000 & 236000 & -1.98 \\
Motor bogie frame & 3306 & 1578 & 2772 & 4200 & -0.5 \\
Trailer bogie frame & 3122 & 1647 & 3453 & 5011 & -0.5 \\
Wheelset & 2091 & 1073 & 120 & 1073 & -0.425 \\
\hline
\end{tabular}

Table 3

Main linear stiffness properties of the ALn 501 "Minuetto".

\begin{tabular}{lll}
\hline Primary suspension & Flexicoil $k_{z}$ & $9.01 \mathrm{E}+05 \mathrm{~N} / \mathrm{m}$ \\
& Flexicoil $k_{x}, k_{y}$ & $1.26 \mathrm{E}+06 \mathrm{~N} / \mathrm{m}$ \\
& Sutuco bushing $k_{x}$ & $2.0 \mathrm{E}+07 \mathrm{~N} / \mathrm{m}$ \\
& Sutuco bushing $k_{y}$ & $1.5 \mathrm{E}+07 \mathrm{~N} / \mathrm{m}$ \\
& Airspring $k_{z}$ & $3.98 \mathrm{E}+05 \mathrm{~N} / \mathrm{m}$ \\
Secondary suspension & Airspring $k_{x}, k_{y}$ & $1.2 \mathrm{E}+05 \mathrm{~N} / \mathrm{m}$ \\
& Anti-roll bar $k_{\alpha}$ & $2.6 \mathrm{E}+06 \mathrm{Nm} / \mathrm{rad}$ \\
& Bushing $k_{x}, k_{z}$ & $7.24 \mathrm{E}+07 \mathrm{~N} / \mathrm{m}$ \\
Coach connection & Bushing $k_{y}$ & $5.2 \mathrm{E}+06 \mathrm{~N} / \mathrm{m}$ \\
\hline
\end{tabular}

The secondary stage (see Fig. 2c) is formed by pneumatic suspensions and it comprises the following elements:

- two air springs;

- six non-linear dampers (lateral, vertical and anti-yaw dampers);

- one non-linear traction rod;

- the roll bar (not visible in the figure);

- two non-linear lateral bumpstops.

The main linear properties of the suspensions are shown in Table 3, while the non-linear characteristics of the vertical and

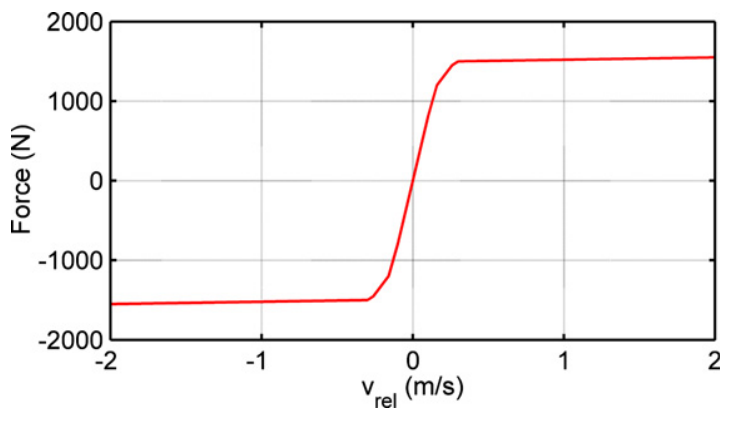

Fig. 3. Nonlinear characteristics: vertical damping of the primary suspension stage.

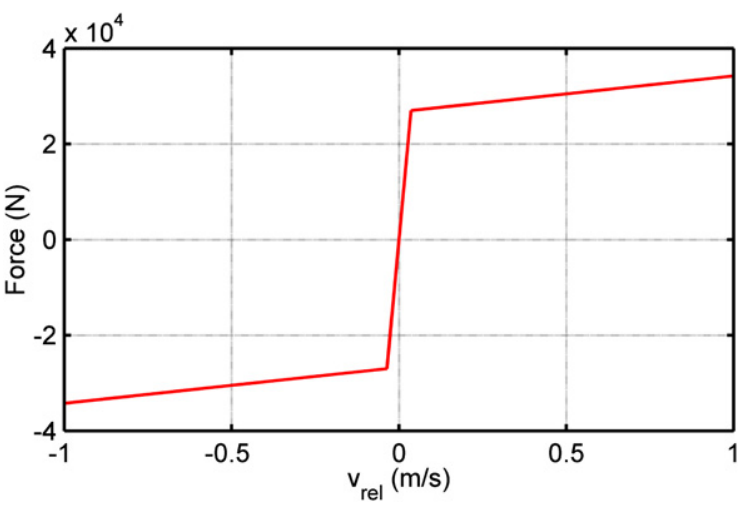

Fig. 4. Nonlinear characteristics: anti-yaw damping of the secondary suspension stage.

anti-yaw dampers are illustrated, respectively, in Figs. 3 and 4 given as a function of the relative velocities.

In this research activity a specifically developed 3D global contact model has been employed to improve reliability and 
accuracy of the contact points detection, compared to those given by the Simpack Rail contact model. The adopted contact model is based on a two step procedure; at the first step the contact points number and positions are determined through an innovative algorithm designed and validated by the authors [2-4]. During the second step, for each detected contact point, the global contact forces are evaluated using Hertz's and Kalker's global theories $[5,6]$.

The new contact points detection algorithm is a fully 3D model that takes into account all the six relative degrees of freedom (DOF) characterizing the wheel-rail interaction and it is able to support generic railway tracks and generic wheel and rail profiles. It also enables a general and accurate treatment of the multiple contact without introducing simplifying assumptions on the problem geometry and kinematics or limits on the number of contact points detected. The algorithm is characterized by a high numerical efficiency that allows the online implementation within the commercial multibody software (Simpack-Rail, Adams-Rail) without discrete look-up tables (LUT) with saved pre-calculated values of contact parameters.

\section{The wear model}

The current section deals with the description of the three phases constituting the wear model: the local contact model, the computation of the amount of worn material (assuming dry contact conditions) and the wheel profile update.

\subsection{The local contact model}

The inputs of the wear model are the global contact parameters estimated by the vehicle model. Since a local wear computation is required, the global contact parameters need to be post-processed and this can be achieved with the simplified Kalker's theory implemented in the FASTSIM algorithm. This theory starts from the global creepages $(\boldsymbol{\varepsilon})$, the normal and tangential global forces $\left(\boldsymbol{N}^{\boldsymbol{r}}, \mathbf{T}_{\mathbf{x}}^{\mathbf{r}}, \mathbf{T}_{\mathbf{y}}^{\mathbf{r}}\right)$, the contact patch dimensions $(a, b)$ and the material properties to compute the local distribution of normal $p_{n}$ and tangential $\mathbf{p}_{t}$ stresses and local creepages $\mathbf{s}$ across the wheel/rail contact area. For a more detailed description of the FASTSIM algorithm one can refers to the literature [9].

\subsection{The wear computation}

The distribution of worn material on wheel profile due to wear (assuming dry contact conditions) is evaluated by means of a wear experimental function, based on a law that relates the energy dissipated in the wheel-rail contact patch with the amount of worn material $[7,8]$.

The adopted wear function uses local contact normal $p_{n}$ and tangential $\mathbf{p}_{t}$ stresses, creepages $\mathbf{s}$ and the vehicle velocity $V$ as the input to compute directly the specific volume of worn material $\delta_{p^{j k}(t)}(x, y)\left(\mathrm{mm}^{3} / \mathrm{mm} \mathrm{mm}^{2}\right)$ related to the $i$-th contact point $P_{w i}^{j k}(t)^{w i}$ on the $j$-th wheel relative to the $k$-th dynamical simulation for unit of distance travelled by the vehicle (expressed in $\mathrm{m}$ ) and for unit of surface (expressed in $\mathrm{mm}$ ). More specifically, local contact stresses and creepages are used to evaluate the wear index $I_{W}$ (expressed in $\mathrm{N} / \mathrm{mm}^{2}$ ), which represents the frictional power developed by the tangential contact pressures:

$I_{W}=\frac{\mathbf{p}_{t} \bullet \mathbf{s}}{V}$.

This index can be correlated with the wear rate $K_{W}$ which represents the mass of removed material (expressed in $\mu \mathrm{g} / \mathrm{m} \mathrm{m}^{2}$ ) for unit of distance travelled by the vehicle and for unit of surface.
The correlation is based on real data available in literature [7], which have been acquired from experimental wear tests carried out in the case of metal to metal contact with dry surfaces using a twin disc test arrangement.

The experimental relationship between $K_{W}$ and $I_{W}$ chosen for the development of the present wear model is described by the following equation:

$K_{W}\left(I_{W}\right)= \begin{cases}5.3 * I_{W} & I_{W}<10.4, \\ 55.12 & 10.4 \leq I_{W} \leq 77.2, \\ 61.9 * I_{W}-4778.68 & I_{W}>77.2 .\end{cases}$

Once the wear rate $K_{W}\left(I_{W}\right)$ has been computed, the corresponding specific volume of worn material (for unit of distance travelled by the vehicle and for unit of surface) can be calculated as follows (expressed in $\mathrm{mm}^{3} / \mathrm{m} \mathrm{mm}^{2}$ ):

$\delta_{P_{w i}^{j k}}(t)(x, y)=K_{W}\left(I_{W}\right) \frac{1}{\rho}$,

where $\rho$ represents the material density (expressed in $\mathrm{kg} / \mathrm{m}^{3}$ ).

\subsection{Profile update and smoothing}

After obtaining the amount of worn material, wheel profile need to be updated and then it can be used as the input of the next dynamic simulation. The new profile, denoted by $w_{n}\left(y_{w}\right)$, is computed from the old one $w_{o}\left(y_{w}\right)$ and from all the calculated distributions $\delta_{p^{i k}(t)}(x, y)$ of worn material through an appropriate set of numericail procedure that defines the update strategy. The update strategy is also applied with the aim of reducing the numerical noise characterizing the distribution $\delta_{p^{j k}(t)}(x, y)$ that can generate problems to the global contact model because of the presence of non-physical alterations in new profiles.

Another issue to be provided from the update procedure is the average of the worn material distributions. In fact, according to Trenitalia and RFI requirements, the output of the wear model must be a single profile; hence the evaluated distribution $\delta_{p_{w i}^{j k}(t)}(x, y)$ needs to be mediate.

The whole numerical procedures that compute the new profiles can be summed up in the following steps:

1. Longitudinal integration:

$$
\frac{1}{2 \pi w\left(y_{w i}^{j k}\right)} \int_{-a(y)}^{+a(y)} \delta_{p_{w i}^{j k}(t)}(x, y) d x=\delta_{p_{w i}^{j k}}^{t o t}(t)
$$

the previous integration provides the mean value of wheel worn material expressed in $\mathrm{mm}^{3} / \mathrm{m} \mathrm{mm}^{2}$. More specifically the operation sums in the longitudinal direction all the wheel wear contributions inside the contact path and distributes the resulting quantity along the wheel circumference of length $2 \pi w\left(y_{w i}^{j k}\right)$.

2. Time integration:

$\int_{T_{i}}^{T_{e}} \delta_{P_{w i}^{j k}(t)}^{t o t}(y) V(t) d t \approx \int_{T_{i}}^{T_{e}} \delta_{P_{w i}^{j k}(t)}^{t o t}\left(S_{w}-S_{w i}^{c j k}(t)\right) V(t) d t=\Delta_{P_{w i}^{j k}}\left(s_{w}\right)$,

where the natural abscissa $s_{w}$ relative to the curve $w\left(y_{w}\right)$ has been introduced. The following relations locally hold (see Fig. 5a):

$y \approx s_{w}-s_{w i}^{c j k}(t), \quad w\left(y_{w}\right)=w\left(y_{w}\left(s_{w}\right)\right)=\tilde{w}\left(s_{w}\right)$.

The natural abscissa of the contact point $s_{w i}^{c j k}$ can be evaluated starting from its position $P_{w i}^{j k}$. The integration (5) sums all the wear contributions relative to the dynamic simulation and gives 


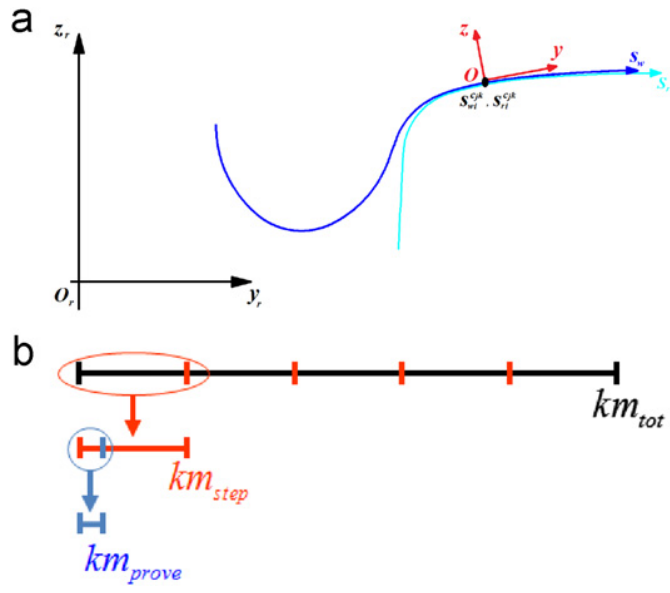

Fig. 5. Wear model: (a) normal abscissa for the wheel and rail profile, (b) discretization of the total mileage.

as output the depth of worn material due to the considered contact point $\Delta_{p^{j k}}\left(s_{w}\right)$ in $\mathrm{mm}=\mathrm{mm}^{3} / \mathrm{mm}^{2}$.

3. Sum on the contaict points:

$\sum_{i=1}^{N_{P D C}} \Delta_{P_{w i}^{j k}}\left(s_{w}\right)=\Delta_{j k}^{w}\left(s_{w}\right)$,

where $N_{P D C}$ represents the maximum number of contact points that can be considered for each single wheel. The output $\Delta_{j k}^{w}\left(s_{w}\right)$ is the removed material of the $j$-th wheel during the $k$-th simulation. The number of active contact points changes during the simulation but it is usually less than $N_{P D C}$; thus, the amount of worn material due to non-active contact points is automatically set equal to zero.

4. Average on the vehicle wheels and on the dynamic simulations:

$\sum_{k=1}^{N_{c}} p_{k} \frac{1}{N_{w}} \sum_{j=1}^{N_{w}} \Delta_{j k}^{w}\left(s_{w}\right)=\bar{\Delta}^{w}\left(s_{w}\right)$

where $N_{w}$ is the number of vehicle wheels while the $p_{k}$, $1 \leq k \leq N_{c}, \sum_{k=1}^{N_{c}} p_{k}=1$ are the statistical coefficients related to the various dynamic simulations. These coefficients have been established by the statistical analysis as will be better explained in the following sections. The average on the number of wheel-rail interactions has to be performed to obtain as output of the wear model a single average profile for the wheel (as required by Trenitalia and RFI).

5. Scaling: Since it normally takes travelled distance of thousands kilometers in order to obtain measurable effects of wheel wear, an appropriate scaling procedure is necessary to reduce the simulated track length with a consequent limitation of the computational effort. The total mileage $\mathrm{km}_{\text {tot }}$ travelled by the vehicle is chosen according to the purpose of the simulations, for example equal to the re-profiling intervals. This mileage is subdivided in steps characterized by a length equal to $\mathrm{km}_{\text {step }}$ and the wheel profile is supposed to be constant within each discrete step (corresponding to a travelled distance equal to $\mathrm{km}_{\text {step }}$ ). The $\mathrm{km}_{\text {step }}$ value represents a distance which is still too long to be simulated in reasonable computational times. However, this can be overcome as follows:

- a linear relationship between the amount of worn material and the travelled distance is supposed to hold only inside the discrete step;

- according to the previous hypotheses a smaller distance $\mathrm{km}_{\text {prove }}$ can be simulated; then the relative amount of wheel worn material can be amplified in order to evaluate the worn material distribution relative to a $\mathrm{km}_{\text {step }}$ travelled distance.

The discrete step definition can be done according to the following two main update strategies: the constant step update strategy, which is characterized by a constant value $\mathrm{km}_{\text {step }}$ of the discrete step and the adaptive step update strategy, wherein the profile is updated when a given threshold $\Delta_{f i x}$ on the maximum value $\Delta_{\max }=\max _{S_{w}} \bar{\Delta}^{w}\left(s_{w}\right)$ of cumulative wear depth is reached; the value $\mathrm{km}_{\text {step }}$ is consequently variable. In the present research the adaptive step approach has been chosen and the discrete step value $\mathrm{km}_{\text {step }}$ can be defined through the following equation:

$k m_{\text {step }}=k m_{\text {prove }} \frac{\Delta_{f i x}}{\Delta_{\max }}$,

where $k m_{\text {prove }}=l_{\text {track }}$ is the total travelled distance simulated during the $N_{c}$ dynamic analysis. The quantity $\Delta_{\max }$ represents the maximum cumulative wear depth while $\Delta_{f i x}$ is the related chosen threshold value and in this work it is equal to $0.1 \mathrm{~mm}$. The scaled amount $\bar{\Delta}^{w} s c\left(s_{w}\right)$ of worn material to be removed by the wheel surface is then given by the following expression:

$\bar{\Delta}^{w} s c\left(s_{w}\right)=\bar{\Delta}\left(s_{w}\right) \frac{\Delta_{f i x}}{\Delta_{\max }}$

6. Smoothing of the worn material:

$\Im\left[\bar{\Delta}^{w} s c\left(s_{w}\right)\right]=\bar{\Delta}_{s m}^{w} s c\left(s_{w}\right)$

the numerical noise and short wavelengths without physical meanings that affect the worn material distribution can be passed to the new wheel profile $\tilde{w}_{n}\left(s_{w}\right)$ with consequent problems raising in the global contact model. Hence an appropriate smoothing of the worn material distributions is required and this is achieved by means of a first-order discrete filter (i.e. a moving average filter with window size equal to $1-5 \%$ of the total number of points in which the profiles are discretized); obviously the discrete filter has to conserve the mass.

7. Profile update:

$$
\left(\begin{array}{c}
y_{w}\left(s_{w}\right) \\
\tilde{w}_{o}\left(s_{w}\right)
\end{array}\right)-\bar{\Delta}_{s m}^{w} s c\left(s_{w}\right) \mathbf{n}_{w}^{r} \stackrel{\text { re-parameterization }}{\longrightarrow}\left(\begin{array}{c}
y_{w}\left(s_{w}\right) \\
\tilde{w}_{n}\left(s_{w}\right)
\end{array}\right)
$$

the last step of the procedure consists in the determination of the new wheel profile $\tilde{w}_{n}(s)=w_{n}(y)$ starting from the old one $\tilde{w}_{o}(s)=w_{o}(y)$. Due to the fact that the removal of material occurs in the normal direction to the profile ( $\mathbf{n}_{w}^{r}$ is the outgoing unit vector for the wheel) once the quantity $\bar{\Delta}_{s m}^{w} s c\left(s_{w}\right)$ has been removed a re-parameterization of the profile must be performed to get curve parameterized by means of the curvilinear abscissa.

\section{Setting-up of the Minuetto virtual track}

The present section is a brief overview on the procedure used in deriving a significant statistical track description, an essential task to make possible and rationalize the approach and the simulation work on a complex railway line.

In the present work the statistical approach has been exploited to draw up the mean line of the Minuetto train. This mean line had to be a significant and equivalent synthesis, in a statistical sense, of the whole set of tracks in Italian railways on which the train composition operates every day. The same strategy has also been used in drawing up a virtual track of the Aosta-Pre Saint Didier line aimed at the model validation via comparison with the available experimental results [4].

The basic idea is to substitute the simulation on the whole track with an equivalent set of simulations on short curved tracks 
(see Table 4). More precisely, the steps performed to get the statistical representation were the following:

- a set of radius curve intervals characterized by a minimum $R_{\min }$ and a maximum $R_{\max }$ were identified analysing the database provided by RFI;

- each of these intervals was furthermore divided in superelevation subclasses, each of them with its own $h_{\min }$ and $h_{\max }$;

Table 4

The Minuetto virtual track.

\begin{tabular}{|c|c|c|c|c|c|c|}
\hline$R_{m}(\mathrm{~m})$ & $R_{M}(\mathrm{~m})$ & $R_{r}(\mathrm{~m})$ & $h_{\text {range }}(\mathrm{mm})$ & $h_{r}(\mathrm{~mm})$ & $V_{r}\left(\mathrm{~km} \mathrm{~h}^{-1}\right)$ & $p_{k}(\%)$ \\
\hline \multirow[t]{2}{*}{250} & 278 & 263 & $90-120$ & 90 & 65 & 1.90 \\
\hline & & & $130-160$ & 160 & 75 & 4.21 \\
\hline \multirow[t]{2}{*}{278} & 313 & 294 & $90-120$ & 90 & 70 & 1.11 \\
\hline & & & $130-160$ & 160 & 80 & 1.62 \\
\hline \multirow[t]{2}{*}{313} & 357 & 333 & $90-120$ & 90 & 70 & 0.44 \\
\hline & & & $130-160$ & 140 & 80 & 1.24 \\
\hline \multirow[t]{3}{*}{357} & 417 & 385 & $50-80$ & 50 & 70 & 0.80 \\
\hline & & & $90-120$ & 120 & 80 & 1.33 \\
\hline & & & $130-160$ & 150 & 90 & 4.17 \\
\hline \multirow[t]{3}{*}{417} & 500 & 455 & $50-80$ & 80 & 70 & 1.44 \\
\hline & & & $90-120$ & 100 & 80 & 4.72 \\
\hline & & & $130-160$ & 130 & 90 & 1.29 \\
\hline \multirow[t]{4}{*}{500} & 625 & 556 & $10-40$ & 10 & 70 & 0.14 \\
\hline & & & $50-80$ & 80 & 80 & 1.52 \\
\hline & & & $90-120$ & 90 & 85 & 2.01 \\
\hline & & & $130-160$ & 150 & 110 & 1.46 \\
\hline \multirow[t]{4}{*}{625} & 833 & 714 & $10-40$ & 10 & 70 & 0.09 \\
\hline & & & $50-80$ & 70 & 85 & 1.56 \\
\hline & & & $90-120$ & 90 & 95 & 1.77 \\
\hline & & & $130-160$ & 130 & 115 & 0.78 \\
\hline \multirow[t]{4}{*}{833} & 1250 & 1000 & $10-40$ & 10 & 70 & 1.10 \\
\hline & & & $50-80$ & 50 & 85 & 2.41 \\
\hline & & & $90-120$ & 120 & 130 & 2.16 \\
\hline & & & $130-160$ & 140 & 130 & 0.93 \\
\hline \multirow[t]{5}{*}{1250} & 2500 & 1667 & 0 & 0 & 70 & 0.17 \\
\hline & & & $10-40$ & 30 & 85 & 1.91 \\
\hline & & & $50-80$ & 80 & 130 & 1.68 \\
\hline & & & $90-120$ & 90 & 130 & 0.99 \\
\hline & & & $130-160$ & 150 & 130 & 0.17 \\
\hline \multirow[t]{5}{*}{2500} & 10000 & 5000 & 0 & 0 & 70 & 1.08 \\
\hline & & & $10-40$ & 20 & 120 & 1.21 \\
\hline & & & $50-80$ & 50 & 130 & 0.25 \\
\hline & & & $90-120$ & 100 & 130 & 0.004 \\
\hline & & $\infty$ & & & & 52.3 \\
\hline
\end{tabular}

- for each subclass a representative radius $R_{m}$ was calculated as a weighted average on all the curve radii included in that subclasses, using the length of curve as a weighting factor;

- the correspondent representative superelevation $h$ was chosen as the most frequent superelevation among the values found in that class;

- for each subclass a speed value $V$ was chosen as the minimum value between the $\max$ speed allowable equal to $V_{\max }=$ $70 \mathrm{~km} / \mathrm{h}$ (depending on the radius, the superelevation and vehicle characteristics) and the speed $\tilde{V}$ calculated imposing a non-compensated acceleration of $a_{n c}^{l i m}=0.6 \mathrm{~m} / \mathrm{s}^{2}$ :

$\frac{\tilde{V}^{2}}{R_{c}}-\frac{h}{S} g=a_{n c}^{l i m}, \quad V=\min \left(\tilde{V}, V_{\max }\right)$;

- a weighting factor $p_{k}$ was introduced for each subclass to take into account the frequency of a certain matching radiussuperelevation in the track and to diversify the wear contributions of the different curves;

- the transition lengths of the real track are incorporated in the constant curvature sections next to them (the inner transition is included in the previous constant curvature section while the outer transition is included in the next one), hence the wear is numerically evaluated on curves and straight tracks only.

The statistical approach to the Italian mean line has provided the classification shown in Table 4 , made up of $N_{C}$ different classes ( 33 curves and the straight track). For each one of the $N_{C}$ classes of curves a different constant average worn rail profile provided by RFI, obviously characterized by a different wear between inner and outer side, has been considered (in Fig. 6 are represented the outer rail profiles); particularly the rail profiles have more severe wear if the curve radius decreases because of the worse contact conditions characterizing the wheel-rail pairs.

\section{Description of the proposed wheel profiles}

This section describes the procedures for the design of the two proposed wheel profiles, named CD1 and DR2 wheel profile.

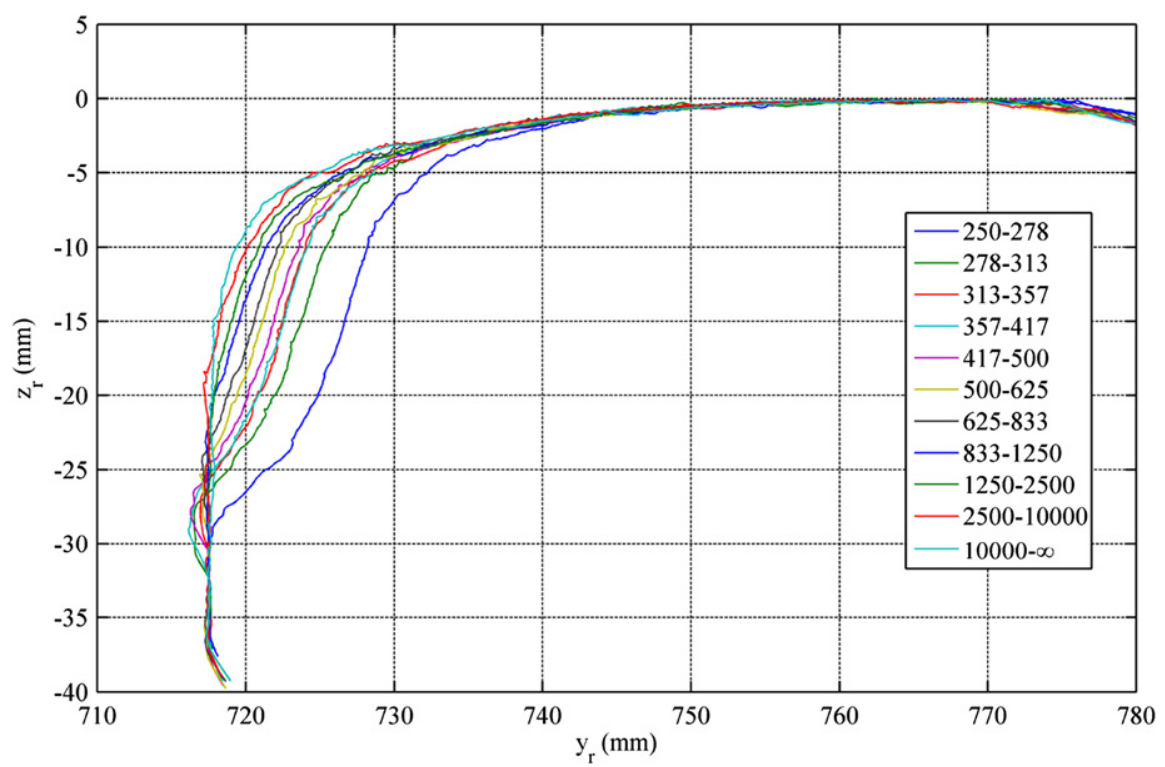

Fig. 6. External worn rail profile of each class (cant of $1 / 20 \mathrm{rad}$ ). 
These procedures have been developed by the authors in collaboration with Trenitalia and RFI [1].

\subsection{CD1 wheel profile}

The CD1 wheel profile has been designed through a discrete procedure in order to achieve the two following goals:

- a better distribution of the contact points in the flange zone in order to improve wear characteristics;

- maintaining the target constant value of the equivalent conicity in a band around the nominal contact point (when the wheelset is in the neutral position).

The adopted nomenclature for the wheel profile construction is shown in Fig. 7. The abscissa $y_{w}$ characterizing the points of the new wheel profile is calculated starting from the abscissa $y_{r}$ of the rail profile which can vary in the range $\left[y_{r 0}, y_{r f}\right]$ discretized with a resolution equal to $0.1 \mathrm{~mm}$ :

$y_{w}=y_{r}\left(s_{y}\right)+s_{y}$,

where $s_{y}$ is the variable representing the horizontal gap between wheel and rail profiles and its value can vary from 0 to the maximum desired horizontal gap $s_{y M A X}$.

The relationship $y_{r}\left(s_{y}\right)$ occurring between the horizontal rail profile coordinate and the horizontal gap can be chosen arbitrarily, but it has to satisfy the following boundary conditions:

$y_{r 0}=y_{r}(0), \quad y_{w 0}=y_{r 0}$,

$y_{r f}=y_{r}\left(s_{y M A X}\right), \quad y_{w f}=y_{r f}+s_{y M A X}$

Considering the purposes previously described, it has been adopted the relationship illustrated in the graphical representation of Fig. 8a. The horizontal part of the graphical illustration represents the purpose in maintaining a constant conicity value in a band around $y_{r}=0$ while the increasing part determines the distribution of the contact points in the flange zone.

Therefore the $z_{w}$ value can be evaluated from the corresponding rail value $z_{r}$ :

$z_{w}=z_{r}\left(y_{r}\left(s_{y}\right)\right)-h\left(s_{y}\right)$,

where the $h\left(s_{y}\right)$ function is defined as

$h\left(s_{y}\right)=-\int_{0}^{s_{y}} \tan \left(\gamma\left(y_{r}\left(s_{y}^{\prime}\right)\right)\right) d s_{y}^{\prime}$.

The variable $\gamma\left(y_{r}\left(s^{\prime}\right)\right)$ represents the contact angle formed by the tangent to the rail profile in $y_{r}\left(s^{\prime}\right)$ and the horizontal plane. The

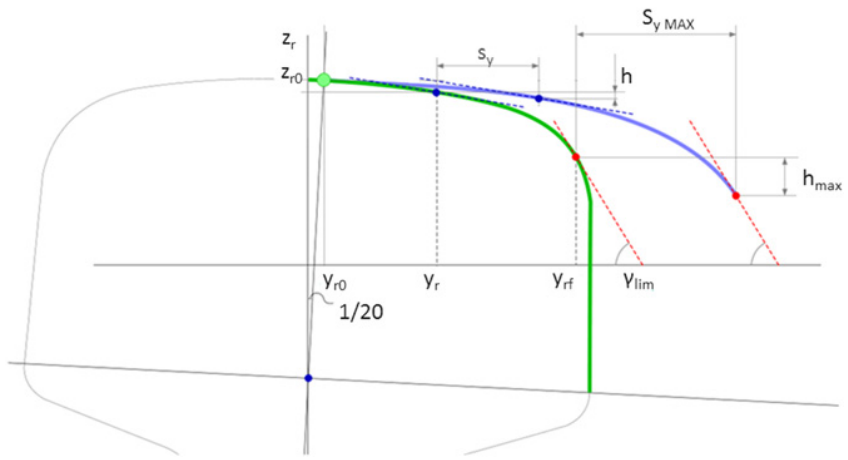

Fig. 7. Design procedure of the CD1 wheel profile. a
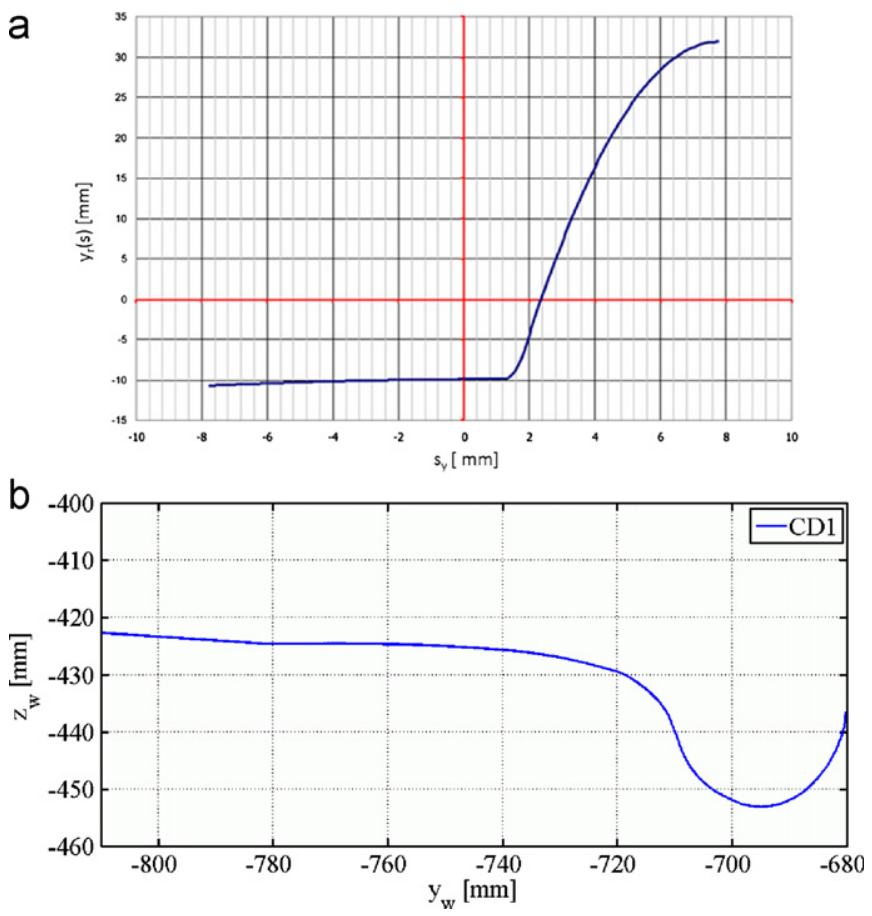

Fig. 8. CD1 wheel profile design: (a) graphical representation of the function $y_{r}\left(s_{y}\right),($ b) CD1 wheel profile.

following boundary conditions hold:

$$
\begin{array}{ll}
h(0)=0, & z_{r 0}=z_{r}\left(y_{r 0}\right), \\
h\left(s_{y M A X}\right)=h_{\max }, & z_{r f}=z_{f}\left(y_{r f}\right), \\
\gamma_{0}=\gamma\left(y_{r 0}\right), & z_{w 0}=z_{r 0}, \\
\gamma_{l i m}=\gamma\left(y_{r f}\right), & z_{w f}=z_{r f}-h_{M A X} .
\end{array}
$$

Fig. 8b illustrates the resulting CD1 wheel profile; it must be noticed that CD1 extremities are designed connecting the resulting profile with the extremity parts of ORE S1002 profile through an interpolation process.

\subsection{DR2 wheel profile}

The design of the DR2 wheel profile aims to guarantee the kinematic characteristics of the original matching formed by ORE S1002 wheel profile and UIC60 rail profile with laying angle $\alpha_{p}$ equal to $1 / 40 \mathrm{rad}$, also with the new matching DR2 wheel profileUIC60 rail profile canted at 1/20 rad. The original matching has been chosen because it is widely common in European railways and it is characterized by good performances in both wear and kinematic behaviour.

The nomenclature adopted for the profile construction, according to the reference systems introduced in the first part of the paper, is shown in Fig. 9 where the apexes $r$ and $w$, respectively, refer to auxiliary and local reference system.

The position of the local reference system origin expressed in the auxiliary reference system is denoted by

$\mathbf{O}_{w}^{r}=\left[\begin{array}{ll}y & z(y)\end{array}\right]^{T}$.

Introducing apexes 1 and 2 to denote, respectively, the right and left wheels, the coordinates of the contact points in the auxiliary reference and the local reference system may be defined as

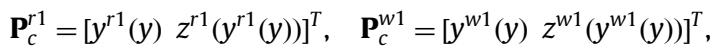

$\mathbf{P}_{c}^{r 2}=\left[\begin{array}{lll}y^{r 2}(y) & z^{r 2}\left(y^{r 2}(y)\right)\end{array}\right]^{T}, \quad \mathbf{P}_{c}^{w 2}=\left[\begin{array}{ll}y^{w 2}(y) & z^{w 2}\left(y^{w 2}(y)\right)\end{array}\right]^{T}$. 


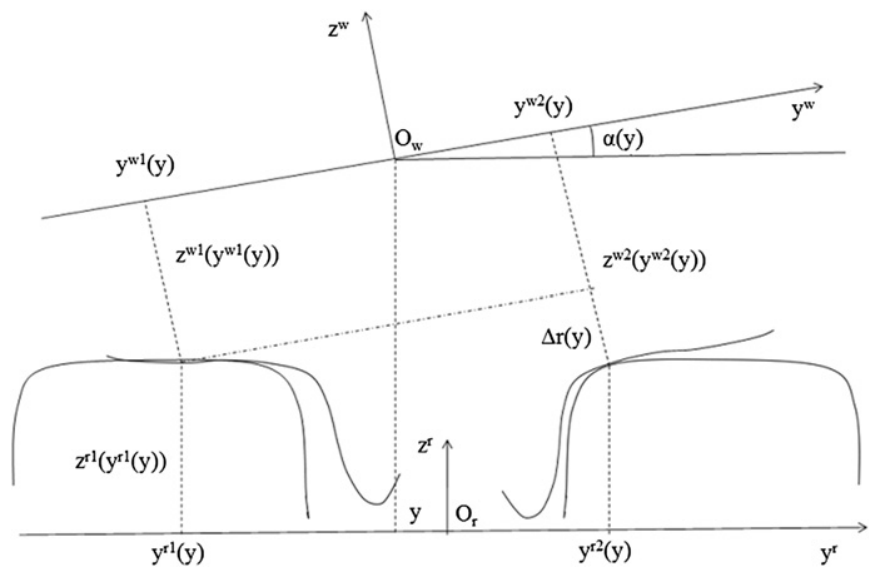

Fig. 9. Adopted nomenclature for DR2 design.

In the present research activity, the purpose in maintaining the kinematic properties of the ORE S1002-UIC60 canted at $1 / 40$ rad matching is achieved by supposing that some variables of the new matching (DR2 wheel profile-UIC60 matching canted at $1 / 20 \mathrm{rad}$ ) remain the same of the original ones. More specifically these variables (all depending on the wheelset lateral displacement $y$ ) are the lateral coordinates $y^{r 1}, y^{r 2}$ of the contact points expressed in the auxiliary reference system, the rail functions $z^{r 1}(\bullet), z^{r 2}(\bullet)$, the vertical coordinate $z(y)$ and the roll angle $\alpha(y)$ of the wheelset.

In the remaining of the paper the variables characterizing the original matching and those referring to the new matching will be, respectively, denoted with the subscripts 40 and 20 .

Consequently the design procedure requires six inputs from the old matching $y_{40}^{r 1}(y), y_{40}^{r 2}(y), \alpha_{40}(y), z_{40}(y), z_{40}^{r 1}(\bullet), z_{40}^{r 2}(\bullet)$ and two further inputs from the new matching $z_{20}^{r 1}(\bullet), z_{20}^{r 2}(\bullet)$. Then, starting from these inputs, the equations describing the coordinate transformation of the contact points between the local and the auxiliary reference system can be written both for the original matching:

$$
\begin{aligned}
& \left(\begin{array}{c}
y_{40}^{r 1}(y) \\
z_{40}^{r 1}\left(y_{40}^{r 1}(y)\right)
\end{array}\right)=\left(\begin{array}{c}
y \\
z_{40}(y)
\end{array}\right)+R\left(\alpha_{40}(y)\right)\left(\begin{array}{c}
y_{40}^{w 1}(y) \\
z_{40}^{w 1}\left(y_{40}^{w 1}(y)\right)
\end{array}\right), \\
& \left(\begin{array}{c}
y_{40}^{r 2}(y) \\
z_{40}^{r 2}\left(y_{40}^{r 2}(y)\right)
\end{array}\right)=\left(\begin{array}{c}
y \\
z_{40}(y)
\end{array}\right)+R\left(\alpha_{40}(y)\right)\left(\begin{array}{c}
y_{40}^{w 2}(y) \\
z_{40}^{w 2}\left(y_{40}^{w 2}(y)\right)
\end{array}\right),
\end{aligned}
$$

and for the new matching:

$$
\begin{aligned}
& \left(\begin{array}{c}
y_{40}^{r 1}(y) \\
z_{20}^{r 1}\left(y_{40}^{r 1}(y)\right)
\end{array}\right)=\left(\begin{array}{c}
y \\
z_{40}(y)
\end{array}\right)+R\left(\alpha_{40}(y)\right)\left(\begin{array}{c}
y_{20}^{w 1}(y) \\
z_{20}^{w 1}\left(y_{20}^{w 1}(y)\right)
\end{array}\right), \\
& \left(\begin{array}{c}
y_{40}^{r 2}(y) \\
z_{20}^{r 2}\left(y_{40}^{r 2}(y)\right)
\end{array}\right)=\left(\begin{array}{c}
y \\
z_{40}(y)
\end{array}\right)+R\left(\alpha_{40}(y)\right)\left(\begin{array}{c}
y_{20}^{w 2}(y) \\
z_{20}^{w 2}\left(y_{20}^{w 2}(y)\right)
\end{array}\right),
\end{aligned}
$$

where the wheelset lateral displacement value $y$ is bounded in the range $\left[-y_{M}, y_{M}\right]$. The outputs of the design procedure that characterize the new wheel profile are the lateral $y_{20}^{w 1}(y), y_{20}^{w 2}(y)$ and vertical $z_{20}^{w 1}\left(y_{20}^{w 1}(y)\right), z_{20}^{w 2}\left(y_{20}^{w 2}(y)\right)$ coordinates of the contact points of the new wheel profile in the local reference system, directly obtained from Eqs. (23) and (24). The design procedure is performed in a discrete way for every $y$ value of the discretized range $\left[-y_{M}, y_{M}\right]$ (with a resolution equal to $0.1 \mathrm{~mm}$ ).

It should be noticed that the resulting profile is characterized by holes (see Fig. 10a) that are the regions where there is not any computed contact point. In the present procedure these regions have been filled fitting the computed points with spline functions and the resulting wheel profile, named DR1, is illustrated in Fig. 10b.

The geometrical wheel/rail contact characteristics are ruled by the rolling radii difference, (the difference between rolling radii of the right and the left wheels for each lateral displacement $y$ ) defined through the following expressions, respectively, valid for the original (ORE S1002 wheel profile and UIC60 canted at $1 / 40 \mathrm{rad}$ ) and the resulting matching (Fig. 11):

$\Delta r_{40}=z_{40}^{w 2}\left(y_{40}^{w 2}(y)\right)-z_{40}^{w 1}\left(y_{40}^{w 1}(y)\right), \quad \Delta r_{20}=z_{20}^{w 2}\left(y_{20}^{w 2}(y)\right)-z_{20}^{w 1}\left(y_{20}^{w 1}(y)\right)$.

The adopted design procedure implies that the rolling radii difference of the output matching is equal to the one characterizing the original matching, disregarding a small estimable variation $e=\Delta r_{20}-\Delta r_{40}$ (see Fig. 12), calculated by means of the following analytical procedure.
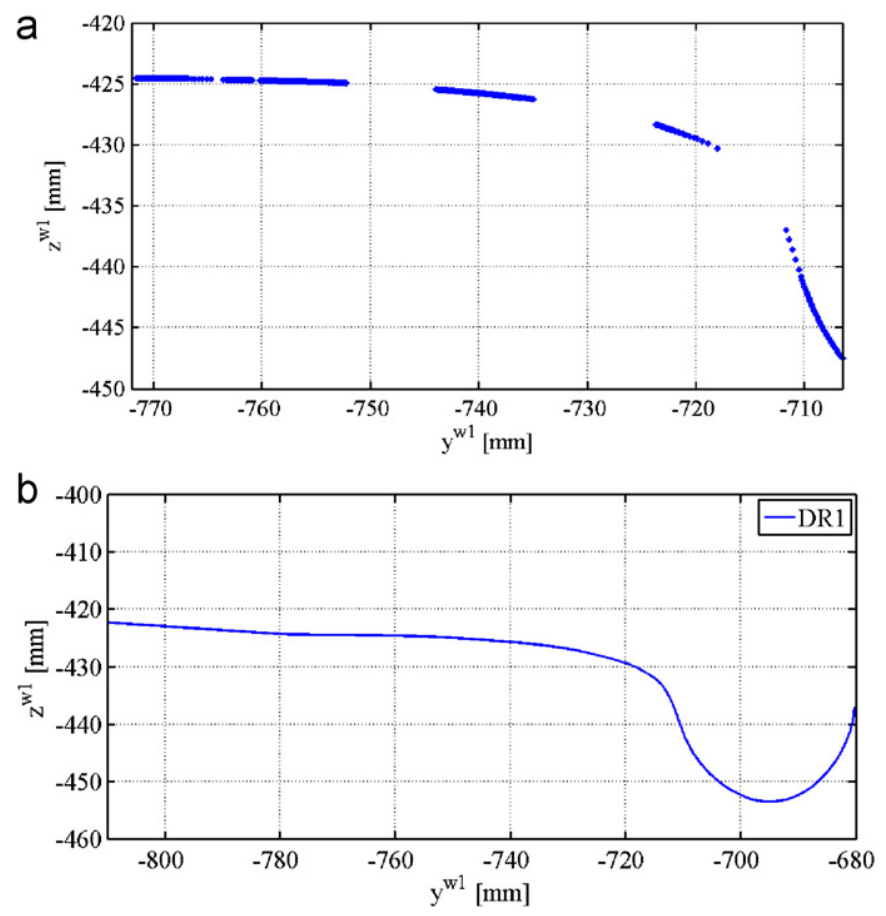

Fig. 10. DR1 wheel profile design: (a) contact points distribution, (b) DR1 wheel profile.

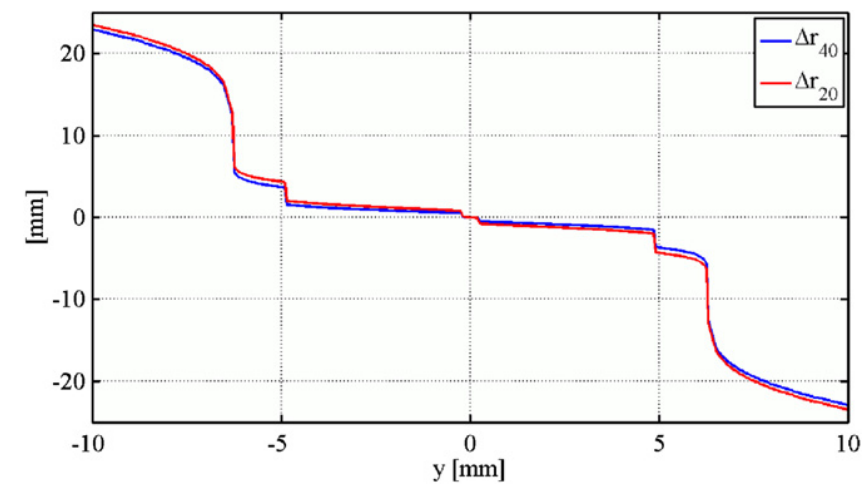

Fig. 11. Rolling radii differences: $\Delta r_{20}=z_{20}^{w 2}\left(y_{20}^{w 2}(y)\right)-z_{20}^{w 1}\left(y_{20}^{w 1}(y)\right)$ relative to the matching DR1-UIC60 canted at $1 / 20$ rad and $\Delta r_{40}=z_{40}^{w 2}\left(y_{40}^{w 2}(y)\right)-z_{40}^{w 1}\left(y_{40}^{w 1}(y)\right)$ relative to the ORE S1002-UIC60 rail canted at 1/40 rad. 


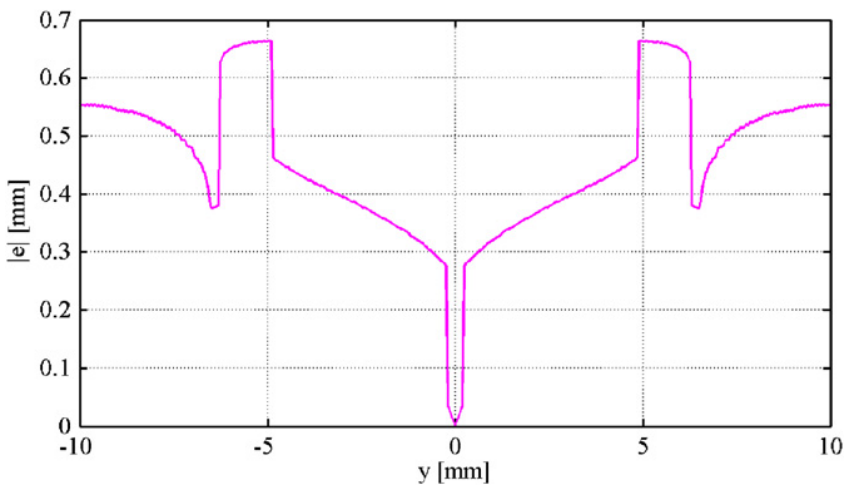

Fig. 12. Absolute value of the error $e$ in rolling radii difference distribution for the DR1-UIC60 canted at 1/20 rad matching.

Subtracting Eq. (21) from Eq. (22) and Eq. (23) from Eq. (24), it leads to the following expressions:

$\left(\begin{array}{c}y_{40}^{r 2}(y)-y_{40}^{r 1}(y) \\ z_{40}^{r 2}\left(y_{40}^{r 2}(y)\right)-z_{40}^{r 1}\left(y_{40}^{r 1}(y)\right)\end{array}\right)=R\left(\alpha_{40}\right)\left(\begin{array}{c}y_{40}^{w 2}(y)-y_{40}^{w 1}(y) \\ \Delta r_{40}\end{array}\right)$,

$\left(\begin{array}{c}y_{40}^{r 2}(y)-y_{40}^{r 1}(y) \\ z_{20}^{r 2}\left(y_{40}^{r 2}(y)\right)-z_{20}^{r 1}\left(y_{40}^{r 1}(y)\right)\end{array}\right)=R\left(\alpha_{40}\right)\left(\begin{array}{c}y_{20}^{w 2}(y)-y_{20}^{w 1}(y) \\ \Delta r_{20}\end{array}\right)$.

Then, subtracting on turn Eq. (26) from Eq. (27) it holds:

$R_{\left(\alpha_{40}\right)}^{T}\left(\begin{array}{c}0 \\ \Delta z_{20}^{r}-\Delta z_{40}^{r}\end{array}\right)=\left(\begin{array}{c}\Delta y_{20}^{r}-\Delta y_{40}^{r} \\ \Delta r_{20}-\Delta r_{40}\end{array}\right)$.

The second component of the previous equation leads to the expression of the rolling radii functions variation between the new and the original matching

$\left(\Delta z_{20}^{r}-\Delta z_{40}^{r}\right) \cos \alpha_{40}=\Delta r_{20}-\Delta r_{40}=e(y)$

as a function of the wheelset lateral displacement where $\Delta z_{20}^{r}=z_{20}^{r 2}\left(y_{40}^{r 2}(y)\right)-z_{20}^{r 1}\left(y_{40}^{r 1}(y)\right)$ and $\Delta z_{40}^{r}=z_{40}^{r 2}\left(y_{40}^{r 2}(y)\right)-z_{40}^{r 1}\left(y_{40}^{r 1}(y)\right)$.

In order to improve the rolling radii difference error between the original matching and DR1 wheel profile-UIC60 canted at $1 / 20$ rad matching, an optimization algorithm has been developed. The basic idea of this algorithm consists in translating the lateral input coordinates $y_{40}^{r 1}(y), y_{40}^{r 2}(y)$ of a certain quantity $k(y)$, evaluated through a minimization process of the rolling radii error for each possible lateral wheelset displacement $y$. The lateral coordinates of the contact points in the auxiliary reference system can be then re-defined as

$y_{40}^{r 1} k=y_{40}^{r 1}+k, \quad y_{40}^{r 2} k=y_{40}^{r 2}+k$,

where the $k$ value is bounded in the range $[-\bar{k},+\bar{k}]=I_{k}$. Therefore the expression of the rolling radii error becomes a function of both $y$ and $k$ values:

$E(y, k)=\cos \alpha_{40}\left(z_{20}^{r 2}\left(y_{40}^{r 2}+k\right)-z_{20}^{r 1}\left(y_{40}^{r 1}+k\right)-z_{40}^{r 2}\left(y_{40}^{r 2}\right)+z_{40}^{r 1}\left(y_{40}^{r 1}\right)\right)$.

Eq. (31) is used as the objective function to find the optimal value $k_{\text {opt }}$ of the translation quantity, which is then defined for each wheelset lateral displacement $y$ as

$k_{\text {opt }}(y)=\arg \min _{k \in I_{k}}|E(y, k)|$.

The optimization process has been performed by discretizing the $I_{k}$ range with a resolution equal to $0.1 \mathrm{~mm}$.

Fig. 13a illustrates the graphical representation of the $k_{o p t}$ value for a determined lateral displacement $y$. It should be noticed that the resulting values are small compared to the characteristic length of the problem. The resulting lateral coordinates of the
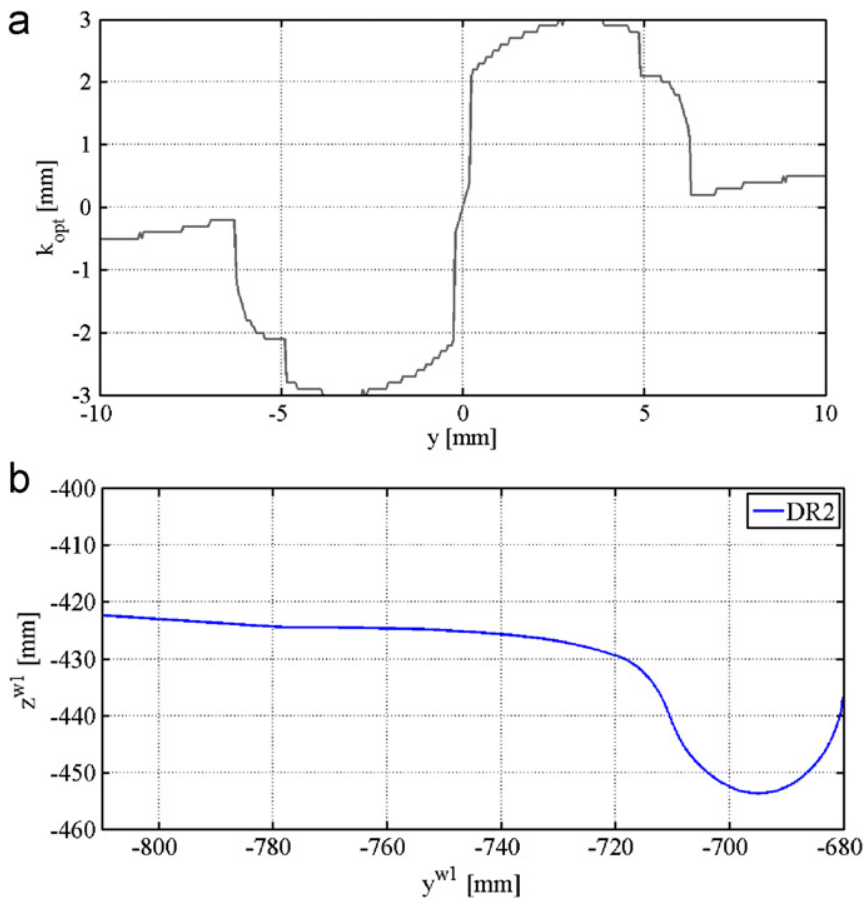

Fig. 13. DR2 wheel profile design: (a) optimal value of the translation quantity $k$, (b) DR2 wheel profile.

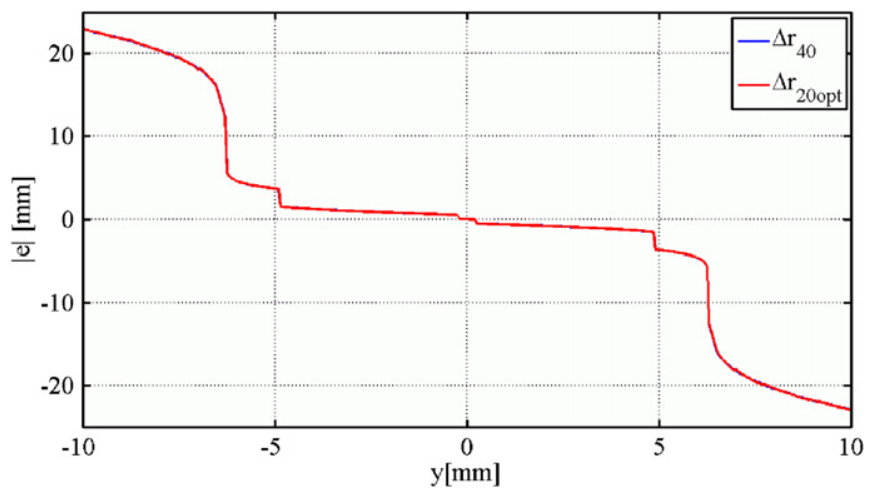

Fig. 14. Rolling radii differences: $\Delta r_{20}=z_{20}^{w 2}\left(y_{20}^{w 2}(y)\right)-z_{20}^{w 1}\left(y_{20}^{w 1}(y)\right)$ relative to the optimized matching DR2-UIC60 canted at $1 / 20 \mathrm{rad}$ and $\Delta r_{40}=z_{40}^{w 2}\left(y_{40}^{w 2}(y)\right)-$ $z_{40}^{w 1}\left(y_{40}^{w 1}(y)\right)$ relative to the ORE S1002-UIC60 rail canted at 1/40 rad.

contact points in the auxiliary reference system are evaluated as $y_{o p t}^{r 1}=y_{40}^{r 1}+k_{o p t}, \quad y_{o p t}^{r 2}=y_{40}^{r 2}+k_{o p t}$.

Through the introduction of these coordinates into Eqs. (23) and (24), the outputs $y_{20}^{w 1}(y), z_{20}^{w 1}\left(y_{20}^{w 1}(y)\right), y_{20}^{w 2}(y), z_{20}^{w 2}\left(y_{20}^{w 2}(y)\right)$ of the optimized wheel profile-UIC60 rail canted at 1/40 rad matching are given by the following expressions:

$\left(\begin{array}{c}y_{o p t}^{r 1}(y) \\ z_{20}^{r 1}\left(y_{o p t}^{r 1}(y)\right)\end{array}\right)=\left(\begin{array}{c}y \\ z_{40}(y)\end{array}\right)+R\left(\alpha_{40}(y)\right)\left(\begin{array}{c}y_{20}^{w 1}(y) \\ z_{20}^{w 1}\left(y_{20}^{w 1}(y)\right)\end{array}\right)$,

$\left(\begin{array}{c}y_{o p t}^{r 2}(y) \\ z_{20}^{r 2}\left(y_{o p t}^{r 2}(y)\right)\end{array}\right)=\left(\begin{array}{c}y \\ z_{40}(y)\end{array}\right)+R\left(\alpha_{40}(y)\right)\left(\begin{array}{c}y_{20}^{w 2}(y) \\ z_{20}^{w 2}\left(y_{20}^{w 2}(y)\right)\end{array}\right)$.

The optimized wheel profile, obtained after the holes fitting procedure and named DR2 wheel profile, is shown in Fig. 13b.

The new rolling radii difference function is compared with the original one in Fig. 14; it shows that the two plots are almost 


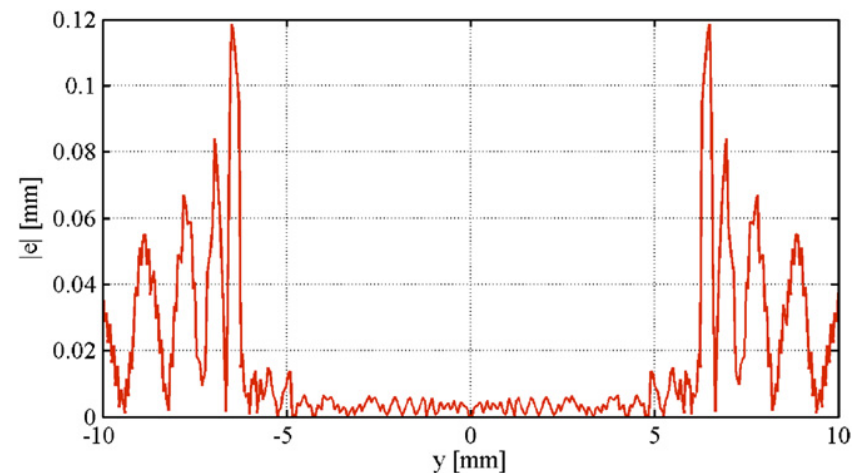

Fig. 15. Absolute value of the error $e$ in rolling radii difference distribution for the DR1-UIC60 canted at 1/20 rad matching.

coincident and that the error (see Fig. 15), which depends on the discretization precision of the range $I_{k}$, is about zero.

The design procedure adopted to define the DR2 discrete wheel profile may be affected by numerical errors coming from different sources such as:

- use of splines in the holes (where there is not a contact point distribution) and of fictitious points at the extremities of the wheel profile (parts of the ORE S1002 have been used);

- subsequent re-interpolations and smooth process of profiles and derivatives of the wheel and the rail;

- since the DR2 wheel profile and UIC60 rail canted at 1/20 rad matching is based on the geometrical properties of the ORE S1002-UIC60 canted at 1/40 rad, it is characterized by the stiffness caused by the conformal contact typical of the original matching.

At the same time, one of the numerical advantages of the procedure consists in the fact that the new DR2 wheel profile is designed without any condition on the derivatives of the profiles; this aspect involves a reduction of the smoothing requirements and does not further increase the ill-conditioning characteristic of the design problem.

\subsection{Comparison between the resulting wheel profiles}

As previously described all the resulting profiles have been designed in order to obtain good kinematic and wear characteristics when matched with the UIC60 rail canted at $1 / 20$ rad. This section deals with the comparison of the CD1, DR1 and DR2 characteristics with those relative to the standard ORE S1002 (optimized to match the UIC60 rail canted at 1/40 rad). Fig. 16 shows the comparison between the resulting CD1, DR1 and DR2 wheel profiles and the original ORE S1002, while in Fig. 18 their relative differences along the vertical coordinates are plotted.

The derivatives of the resulting CD1, DR1 and DR2 wheel profile compared with the derivative of the standard ORE S1002 are illustrated in Fig. 19.

Differences in CD1 and DR2 wheel profiles properties coherently reproduce the fact that they have been produced with two different design aims and procedures. The DR1 and DR2 wheel profiles are instead almost coincident, representing that the DR2 optimization algorithm may improve the DR1 designing procedure which nevertheless, produces itself a wheel profile with good kinematic and wear characteristics. It can be noticed that the new DR1 and DR2-UIC60 canted at 1/20 matching try to reproduce the conformal contact characterizing the original matching ORE S1002-UIC60 canted at 1/40 with coherent vertical translations of the wheel profiles and derivatives in the tread and flange zone.

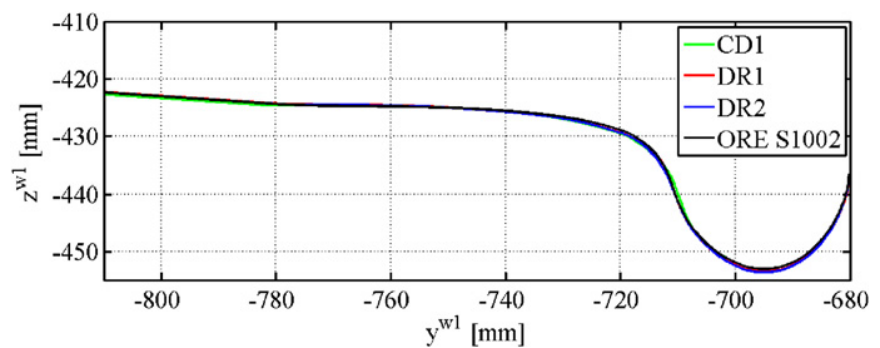

Fig. 16. CD1, DR1, DR2 and ORE S1002 wheel profiles.
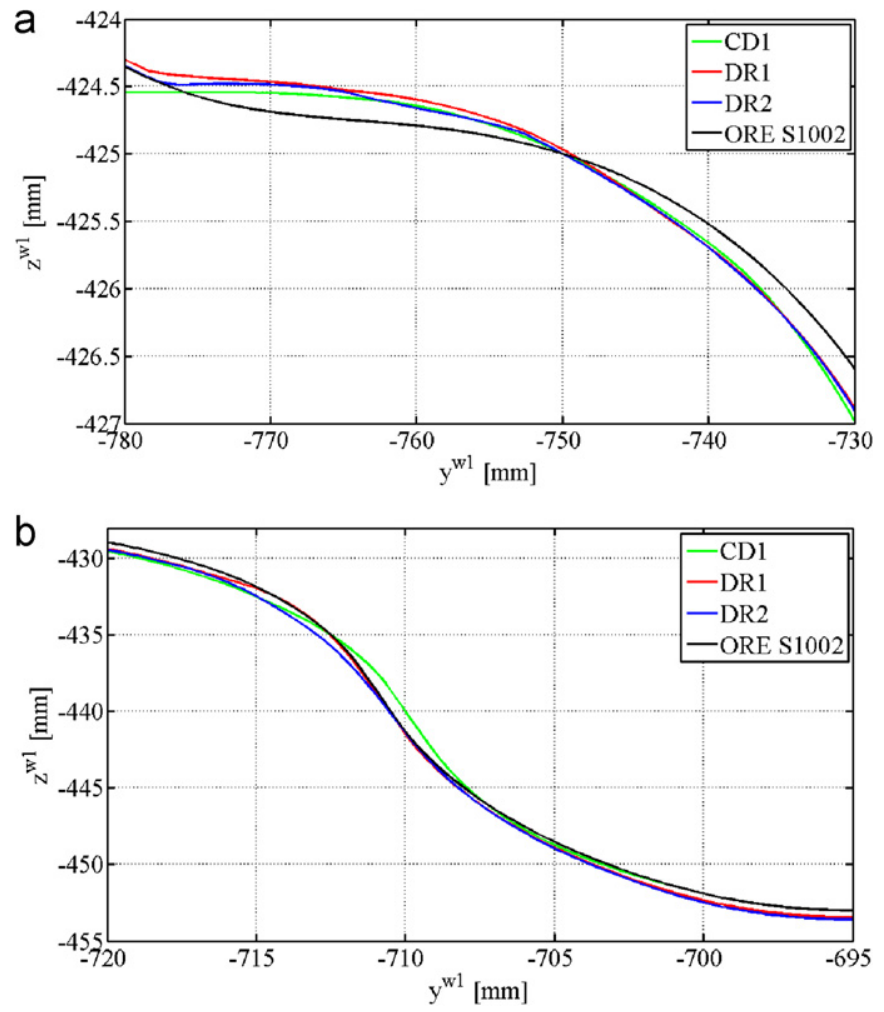

Fig. 17. Comparison of the four wheel profiles: (a) tread zone, (b) flange zone

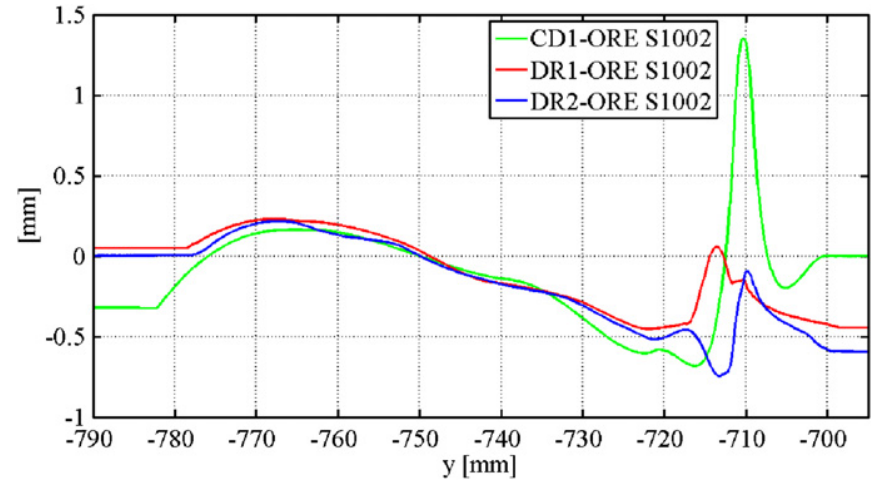

Fig. 18. Vertical differences CD1-ORE S1002, DR1-ORE S1002 and DR2-ORE S1002.

More specifically the points of the new wheel profiles in the tread zone (Fig. 17a) are translated upwards with respect to those characterizing the original ORE S1002 wheel profile, while the points in the flange zone slope downwards (Fig. 17b). 


\section{Wear control parameters}

According to the regulations in force [10], the wear condition in a wheel can be evaluated through the measurement of three particular dimensions (the $q R$ quota, the flange thickness $f T$ and the flange height $f H$, Fig. 20), without a complete detection of the $2 \mathrm{D}$ profile. The three dimensions and the adopted nomenclature are defined as follows (Fig. 20):

- the point P0 on the profile is $70 \mathrm{~mm}$ distant from the internal side of the wheel;

- the point $\mathrm{P} 1$ is $2 \mathrm{~mm}$ above the lowest point $\mathrm{V}$ of the flange on the wheel profile;

- the point P2 is $10 \mathrm{~mm}$ below P0 on the profile;

- the flange thickness $f T$ is defined as the distance between P2 and the internal vertical side of the wheel; the $q R$ is the horizontal distance between $\mathrm{P} 1$ and $\mathrm{P} 0$; the flange height $f H$ is the vertical distance between P0 and V. P0 is also the point where the conventional rolling radius of a wheel is taken.

Because of the way the quotas are defined, they are positive and do not depend on the wheel rolling radius. The values of these parameters are measured periodically in order to decide whether the profile has to be re-turned or not (if it is still possible), considering the maximum or minimum values suggested by the regulations [10]. These limit values are reported in Fig. 20.

As regards their physical meaning, both the flange thickness $f T$ and the flange height $f H$ describe the size of the flange: variations of the first quota are due to the action of wear which progressively reduces the thickness of the flange and its structural resistance, while the rise of the flange height is a measure of the wear on the tread. Conversely, the $q R$ dimension is a shape parameter which quantifies the local conicity on the flange. Although the performance in terms of dynamic behaviour depends on the coupling between the wheel and rail profiles rather than just the
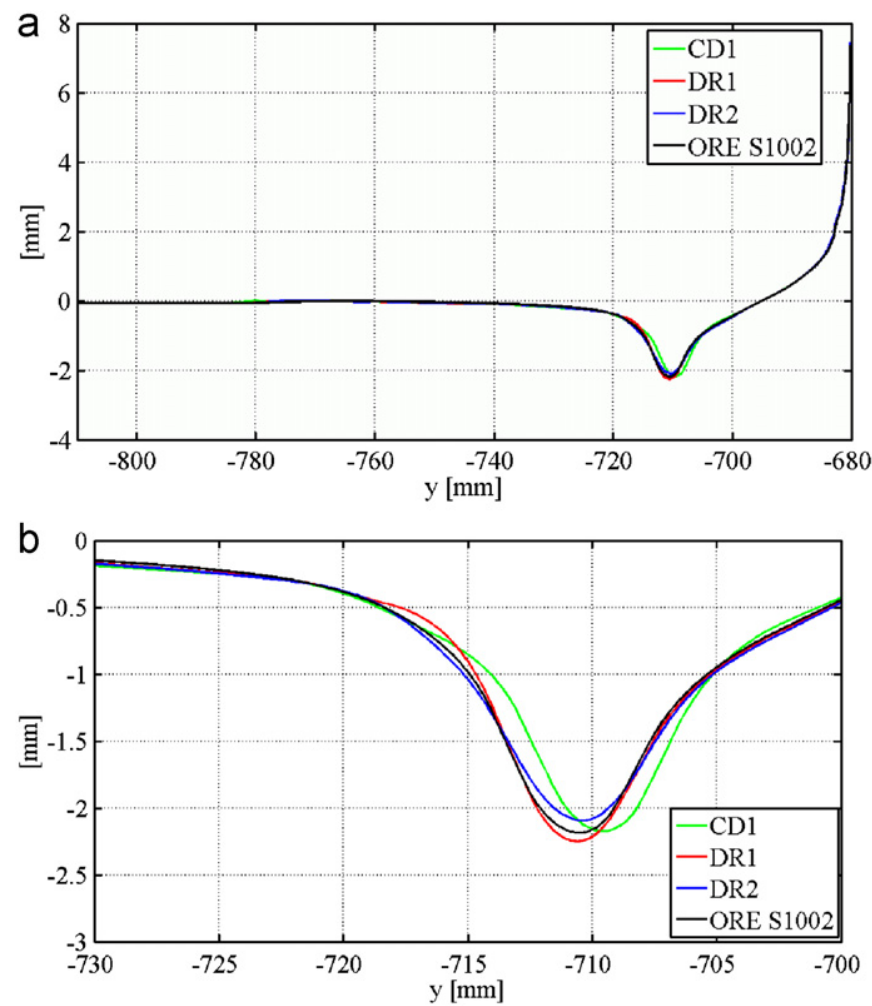

Fig. 19. CD1, DR1, DR2 and ORE S1002 wheel profile derivatives.

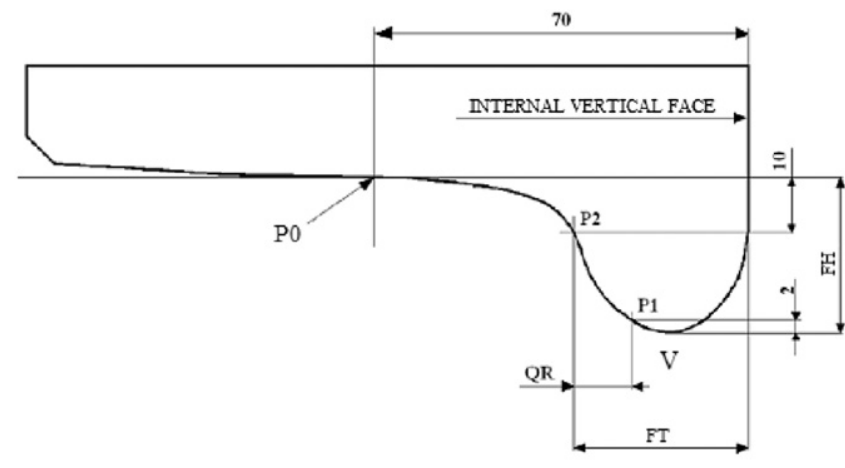

\begin{tabular}{llccc} 
& & $d \leq 630$ & $630<d \leq 760$ & $760<d$ \\
\cline { 2 - 4 }$f H$ & $\min$ & 31.5 & 29.5 & 27.5 \\
& $\max$ & & 36 & \\
\hline
\end{tabular}

\begin{tabular}{ccccc} 
& & $d \leq 760$ & $760<d \leq 840$ & $840<d$ \\
\cline { 2 - 4 }$f T$ & $\min$ & 27.5 & 25 & 22 \\
& $\max$ & \multicolumn{3}{c}{33} \\
\hline
\end{tabular}

$q R \quad \min \quad 6.5$

Fig. 20. Reference dimensions of the wheel profile (left) and limit values in $\mathrm{mm}$ (right) for a wheel having an actual rolling diameter equal to $d$.

wheel profile, the check of the reference quotas aims to guarantee an acceptable running behaviour; in particular, the safety against the hunting in straight track at high speed and the derailment is of fundamental importance. The first phenomenon is enhanced by high values of equivalent conicity at the wheel-rail contact interface, while the second one can occur in case of worn wheel profile characterized by a low flange angle, which reduces, all other things being equal, the maximum allowable lateral force on wheel.

\section{Wear analysis}

In this section the results of the dynamic simulations aimed at a wear evaluation will be presented in order to compare the profiles considered in this study: the standard S1002 and the two innovative profiles (CD1, DR2). For what concerns the resistance to wear, the performance can be assessed by analysing the evolution of three reference dimensions introduced in the previous Section 7.

To this end, Fig. 21 shows the progress of the mean $q R$ dimension for each profile: as it can be seen, the progress of the CD1 and DR2 profiles is slower than that of the S1002; in particular, the best performance is given by the DR2 profile. In fact, assuming a comparison limit equal to $7 \mathrm{~mm}$, which is slightly above than the acceptable threshold value of $6.5 \mathrm{~mm}$ prescribed by the standard [10], the trend of the DR2 shows that the comparison limit is reached with an increase in the covered distance by at least 30\%.

In regarding to the progress of the flange thickness $f T$ depicted in Fig. 22a, the minimum value equal to $22 \mathrm{~mm} \mathrm{[10]} \mathrm{is} \mathrm{reached}$ after covering about $80000 \mathrm{~km}$ when the $\mathrm{S} 1002$ profile is adopted on the Minuetto; differently, with the new profiles the total covered distance can be extend up to $100000 \mathrm{~km}$ and above. Differently from the $q R$, in the reduction of the flange thickness the difference between the performance provided by the CD1 and DR2 profile is about $5 \%$ only.

In regarding to the flange height $f H$, the comparison is depicted in Fig. 22b: this quota usually increases owing to the wear on the 


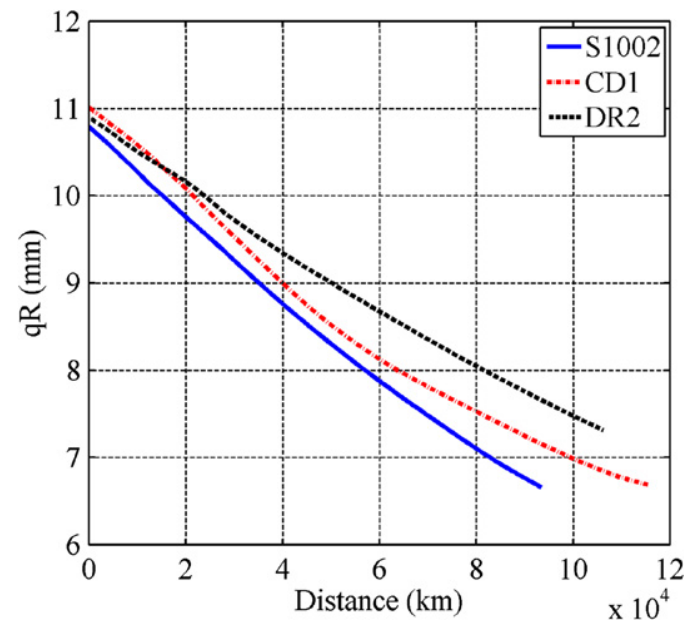

Fig. 21. Progress of the $q R$ dimension: comparison of the wheel profiles.
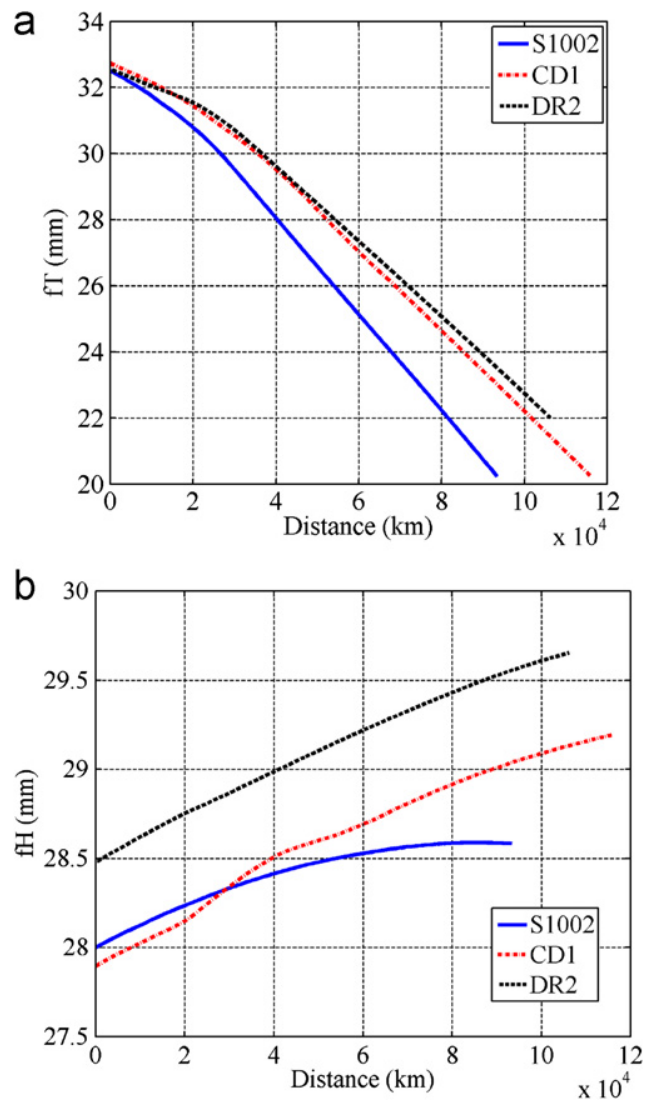

Fig. 22. Comparison of the wheel profiles: (a) progress of the $f T$ dimension, (b) progress of the $f H$ dimension.

tread of the profile. The trend of this dimension for the two innovative wheel profiles is the same, except for an initial offset due to the different strategies with which they were generated. However, generally the flange height progress it is less important than the progress of the other dimensions ( $f T$ and $q R$ ) because does not lead the wheel to a end of life condition.

With respect to the evolution of the wheel shape, the comparison between the initial and the final conditions for the three profiles is presented in Figs. 23-25. The variation in wheel profile is numerically described by means of about 100 procedure steps and besides the worn and the final profile, all the intermediate wheel geometries have also been plotted in the relative figure.

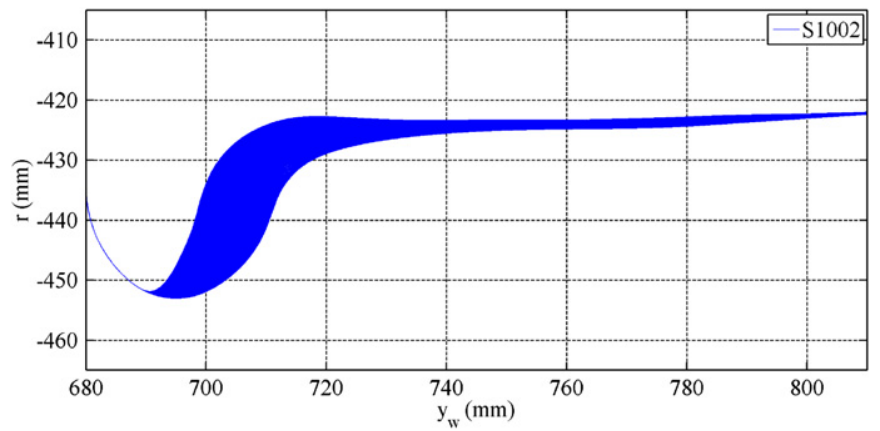

Fig. 23. Evolution of the $S 1002$ wheel profile due to wear.

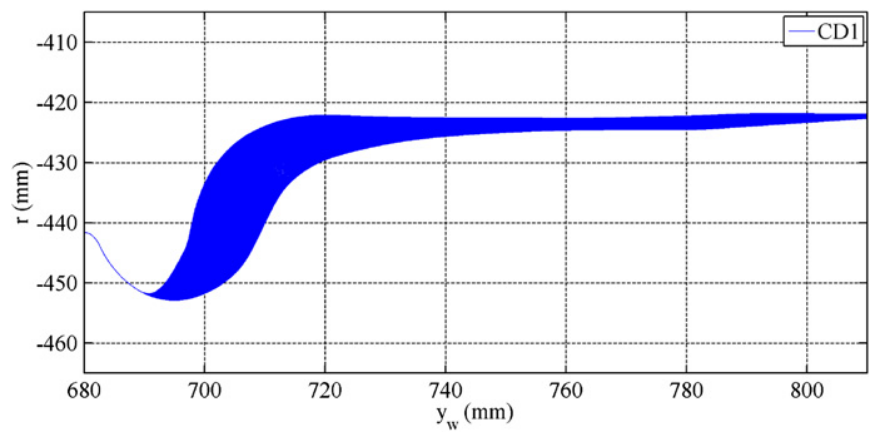

Fig. 24. Evolution of the CD1 wheel profile due to wear.

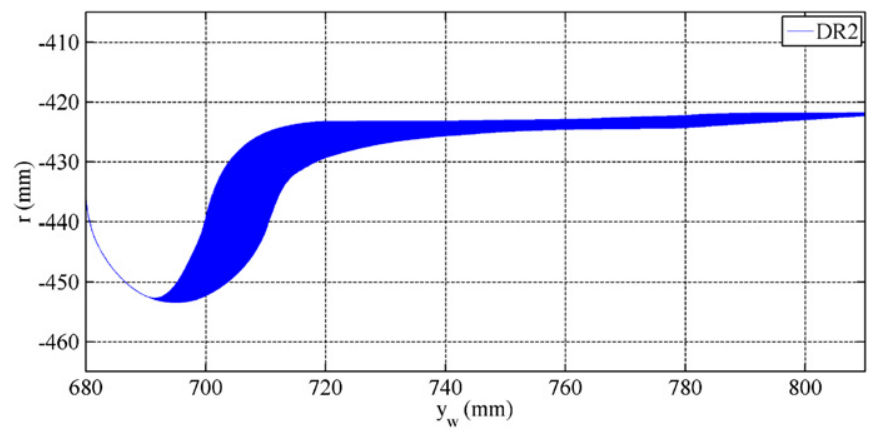

Fig. 25. Evolution of the DR2 wheel profile due to wear.

Since the mean line of the Minuetto comprises a relevant percentage of sharp curves, the wear is mainly located on the flange instead of the tread.

\section{Conclusions}

In this paper the authors have presented a work focused on the development of a mathematical model for the wear evaluation in railway vehicles and on the comparison between the performance provided by different wheel profiles in terms of resistance to wear. More precisely, the standard ORE S1002 wheel profile (matched to the UIC60 rail profile canted at 1/20 rad) widely used on vehicles in service on the Italian railways has been compared with two innovative wheel profiles developed by authors to improve the poor performance of the $\mathrm{S} 1002$ profile with regard to the resistance to wear.

The activity has been performed in collaboration with Trenitalia S.p.A and Rete Ferroviaria Italiana (the administrator of the Italian railway infrastructure) which provided the necessary technical 
and experimental data for the implementation of the whole procedure aimed at wear evaluation.

In particular, the developed architecture is based on the mutual interaction between two main parts. The first one is responsible for the vehicle dynamics and comprises a multibody model of the vehicle under investigation and a global wheel-rail contact model developed by authors in previous works. The second part is a wear model with which the amount and the distribution of material to be removed on wheels is evaluated by exploiting the outputs of the multibody simulation. The entire procedure was validated in a previous work [4] by means of the experimental data, provided by Rete Ferroviaria Italiana, regarding to the Aosta Pre-Saint Didier track and the ALn 501 "Minuetto vehicle".

The developed model has been used in this work for a wear assessment on the ALn 501 Minuetto taking into account the whole Italian railway net in which these vehicles operate. The wear progress has been simulated on an equivalent statistical model of the complex railway, built by consulting the detailed database provided by Rete Ferroviaria Italiana.

The two innovative wheel profiles developed in this activity for the UIC60 rail with a cant of $1 / 20$ rad have proven to work fine as for the resistance to wear if compared with the $\mathrm{S} 1002$ wheel profile. In addition, the kinematic characteristics of these innovative wheelrail coupling are appreciably better than those of the S1002.

Future developments of the present work will be based on the experimental evaluation of the wear on the Minuetto equipped with the new wheel profiles, to verify the response in terms of progress of the reference dimensions. To this end, experimental tests have been scheduled by Trenitalia and will be soon carried out; after choosing a particular track, a few vehicles having the same weekly shift will be equipped with the three profiles taken into account in this work in order to compare their performance both in terms of resistance to wear and running stability.

\section{Acknowledgements}

Authors would like to thank Engg. R. Cheli, G. Grande and R. Desideri of Trenitalia S.p.A. for providing the data relative to the Aln 501 Minuetto vehicle and for their technical support during the whole research activity. A special thanks also goes to the Engg. R. Mele and M. Finocchi of Rete Ferroviaria Italiana for the data relative to the Italian railway lines on which the Minuetto vehicle operates.

\section{References}

[1] P. Toni, Ottimizzazione dei profili delle ruote su binario con posa $1 / 20$, Technical Report, Trenitalia S.p.A., 2010.

[2] J. Auciello, E. Meli, S. Falomi, M. Malvezzi, Dynamic simulation of railway vehicles: wheel/rail contact analysis, Vehicle System Dynamics 47 (2009) 867-899.

[3] E. Meli, S. Falomi, M. Malvezzi, A. Rindi, Determination of wheel-rail contact points with semianalytic methods, Multibody System Dynamics 20 (2008) 327-358.

[4] M. Ignesti, M. Malvezzi, L. Marini, E. Meli, A. Rindi, Development of a wear model for the prediction of wheel and rail profile evolution in railway systems, Wear 284-285 (2012) 1-17.

[5] J.J. Kalker, Three-dimensional Elastic Bodies in Rolling Contact, Kluwer Academic Publishers, Dordrecht, Netherlands, 1990.

[6] H. Hertz, The contact of elastic solids, Journal für die Reine und Angewandte Mathematik 92 (1881) 156-171.

[7] F. Braghin, R. Lewis, R.S. Dwyer-Joyce, S. Bruni, A mathematical model to predict railway wheel profile evolution due to wear, Wear 261 (2006) 1253-1264.

[8] R. Enblom, M. Berg, Simulation of railway wheel profile development due to wear influence of disc braking and contact environment, Wear 258 (2005) 1055-1063.

[9] J.J. Kalker, A fast algorithm for the simplified theory of rolling contact, Vehicle System Dynamics 11 (1982) 1-13.

[10] EN 15313: Railway applications - in-service wheelset operation requirementsin-service and off-vehicle wheelset maintenance, 2010. 